\title{
A Novel Method for the Determination of The Change in Blade Tip Timing Probe Sensing Position Due to Steady Movements
}

DOI:

10.1016/j.ymssp.2019.02.016

\section{Document Version}

Accepted author manuscript

Link to publication record in Manchester Research Explorer

Citation for published version (APA):

Mohamed, M., Bonello, P., \& Russhard, P. (2019). A Novel Method for the Determination of The Change in Blade Tip Timing Probe Sensing Position Due to Steady Movements. Mechanical Systems and Signal Processing, 126, 686-710. https://doi.org/10.1016/j.ymssp.2019.02.016

Published in:

Mechanical Systems and Signal Processing

\section{Citing this paper}

Please note that where the full-text provided on Manchester Research Explorer is the Author Accepted Manuscript or Proof version this may differ from the final Published version. If citing, it is advised that you check and use the publisher's definitive version.

\section{General rights}

Copyright and moral rights for the publications made accessible in the Research Explorer are retained by the authors and/or other copyright owners and it is a condition of accessing publications that users recognise and abide by the legal requirements associated with these rights.

\section{Takedown policy}

If you believe that this document breaches copyright please refer to the University of Manchester's Takedown Procedures [http://man.ac.uk/04Y6Bo] or contact uml.scholarlycommunications@manchester.ac.uk providing relevant details, so we can investigate your claim.

\section{OPEN ACCESS}


1 A Novel Method for the Determination of The Change in Blade Tip

2 Timing Probe Sensing Position Due to Steady Movements

3 Mohamed Mohamed $^{\mathrm{a}}$, Philip Bonello ${ }^{\mathrm{a}}$, Peter Russhard ${ }^{\mathrm{b}}$

4

${ }^{a}$ School of Mechanical, Aerospace and Civil Engineering, University of Manchester, Manchester, United Kingdom

${ }^{\mathrm{b}}$ EMTD Ltd, United Kingdom

\section{ABSTRACT}

The correlation of blade tip timing (BTT) measurements against strain gauge (SG) measurements and finite element (FE) predictions includes a number of uncertainties. One of the main ones is the steady movement of the blades (i.e. change in their mean position and orientation). This causes the sensing positions of the probes relative to a blade tip to deviate from their intended (nominal) positions, leading to deceptive results for the BTT amplitude and the corresponding stress levels. Such movements are caused by variations in static loading conditions (thermal and pressure) associated with changes in the operating speed. A novel method is introduced for the determination of three basic types of blade tip steady movements: axial; lean; untwist. The method relies on linking the shift in the averages of the BTT data to a number of geometrical relations, depending on the type of movement. Not more than two probes (to be placed at different axial positions) are needed to measure all three types of movement. The method is validated by simulations using a novel BTT simulator, and by measurements from both a test rig and real engine tests. The validated results demonstrate the great potential of the method for practical applications.

\section{Keywords}

Blade Tip Timing; Measurement systems; Bladed assembly mechanics; Finite Element Modelling 


\section{INTRODUCTION}

2 The severe operating environment in turbomachinery caused by high speed, temperature and pressure, produces high dynamic stresses in their bladed assemblies. Blade vibration measurements

4 provide the designers of turbomachinery applications with the necessary information to determine the limitations posed by such conditions on the structural integrity of components, particularly in relation to fatigue and endurance limits, and to improve the design accordingly.

Blade tip timing (BTT) is a non-intrusive, non-contact, system for measuring the vibration of the blade tips of rotating bladed assemblies thus enabling the determination of the fatigue limits of such components. This is done by measuring the arrival times of the blade tips at the angular positions of a number of probes during one or more shaft revolutions and comparing these with their expected times of arrival derived from a once-per-revolution probe. The difference between the actual and expected arrival times of a blade at a given probe is a measure of the instantaneous tip displacement over and above the rigid body rotational motion, and such displacements ("BTT data") are processed by a BTT algorithm for frequency, amplitude and phase information. Since BTT measurements refer to the blade tip, their conversion to stress information at critical points on the blade requires prior replace the latter with BTT since it has the following advantages: (a) it can measure the displacements of all blades, whereas with SG only a few blades can be instrumented; (b) there is no interference with the blades; (c) no cables are connected to the rotating parts, thus there is no need

21 for expensive slip rings or telemetry systems required by SGs; (d) the probes used in BTT can sustain harsh operating conditions, unlike SGs, thus reducing costs.

23 Since its inception in the 1960's, BTT has been subjected to many developments with regard to both the instrumentation/data acquisition aspect, and the BTT data analysis algorithms aspect. The most 
1 considerable development of the current BTT technology relating to the data analysis algorithms was

2 started about twenty years ago by the work of Heath [1-3]. This was followed by the Auto-

3 regressive methods of [4-6] and more recently by the multi-frequency sine fitting method by

4 Russhard [7]. The latter method involved the preparation of data to separate synchronous from non-

5 synchronous vibration components, and is currently the standard method used in a leading aeroengine company. More recent improvement has been done by Rigosi et al. [8], who improved the early two-parameter plot method introduced by Heath [2], and the method of Jun Lin et al. [9] for monitoring multi-mode vibration signals. Other methods have been published with little information.

9 like the circumferential Fourier Fit (CFF) method [10], or have developed within industry and kept 10 confidential.

11 The correlation of BTT measurements with FE includes many sources of uncertainty. These can be 12 divided into three groups as follows [11][12]:

- Measurements uncertainties;

- BTT data processing/analysis uncertainties;

- Finite element (FE) model uncertainties.

One of the main sources of BTT measurements uncertainties is the change in the mean position and orientation of the blade tip. The general motion of any blade has three components:

- Rotation as a rigid body about the shaft axis;

- Vibration arising from dynamic forces;

- Steady (non-oscillatory) movements which alter the mean position and orientation of the

22 The latter are caused by variations in operating conditions that are associated with a change in the rotational speed: centrifugal loading, thermal expansion of the rotor, bearing wear, axial float, and 
non-uniform gas loading. The displacement at the blade tip caused by the blade steady movement

can be resolved into three component movements:

- Axial movement, where the direction of movement of the blade tip chord line is perpendicular to the plane of rotation, as shown in Fig. 1a.

- Lean, where the blade tip is moving perpendicular to the blade chord line, as shown in Fig. $1 b$.

- Untwist, where the blade tip is twisting around an axis parallel to the longitudinal axis of the blade, which is also parallel to the plane of rotation as shown in Fig. 1c.

\section{Fig. 1(a,b,c) HERE}

These blade tip movements are illustrated in Fig. 1 in the context of the steady movement/deformation of the whole blade, where the faint colour represents the initial position of the blade. In the case of axial float of the shaft, the consequent shift of the bladed disk assembly translates to an identical shift of the blade tip, as per Fig. 1a. In the case of shaft bow caused by thermal expansion, the blade will tilt. Although Figs. 1a-c do not show a tilted blade, the movements of the tip of a tilted blade are still covered by the basic blade tip movements illustrated in Figs 1a-c i.e. translatory (axial/lean) plus rotational (untwist). This paper is concerned with the steady movement of the blade tip, which results in a change in the point where the BTT measurement is made since the sensing positions of the probes relative to a blade tip will become shifted from their intended (nominal) positions. Such movement is considered as a "DC" error in the measurement [13]. This can have a huge impact on the calibration factor relating tip deflection to stress calculated from a validated FE model of the bladed disk ("blisk"), thus introducing errors in the fatigue limit estimates. Indeed, the new detection position of the probe relative to the tip may coincide with a node (point of minimal vibration) of a certain mode shape, causing the mode to be missed (see Fig. 
$12 \mathrm{a}$ ), or may completely miss the blade in case the probe is placed close to the leading or trailing

2 edges (see Fig. 2b). Both cases in Figs 2a,b lead to a potentially large percentage of error in the

3 calculated stress values [14].

6 Currently available BTT data algorithms are concerned only with the determination of the frequency, amplitude, and phase of the vibration component of the blade tip motion. Some of these can consider the effect of probe offsets (their angular position errors) which are constant i.e. independent of speed (section 3). All current BTT algorithms neglect the effect of steady movements of the blade tip, despite their critical impact on the results. A few research works have attempted to identify and quantify the steady movements of the blades relative to the probes. It is reported in [14] that some preliminary methods have been developed at a leading aero-engine company, requiring the addition of more probes and knowledge of the predicted mode shapes, in order to determine the steady relative movement, but no details have been published. Jousselin [15] proposed a method for the extraction of the shifted measurement position in case of lean, axial shift, or untwist of shrouded turbine blades, in which the blade tip has peculiar design features (two fences) that are not found in either compressor blades or unshrouded turbine blades. These features were exploited for BTT detection purposes since the blade arrival data comprised four different timing data per revolution from the two fences. Different formulas were introduced for the calculation of the steady movement based on the timing data and the geometry of the fences. However, the method was limited to this special type of blade, and was neither validated experimentally nor with simulated data [15].

Hatcher Jr et al. [16] has presented a method of capturing the blade tip using an image capture 23 device simultaneously with the blade tip detection, and then locating the measurement position manually or by image processing. Kominsky [17] has presented a way of calculating the untwist angle by using optical fibres and measuring the intensity of light reflected from the blade. As 
1 observed in [13], the aforementioned methods required the use of additional equipment, thus

2 reducing reliability and increasing costs, or depended on an additional feature on the blade tip, thus

3 restricting the method to particular blade types. What is needed is a method based on BTT

4 technology which can identify and quantify the steady movement, thus tracking changes in the

5 nominal sensing positions, and which retains all the advantages of BTT.

6 In this work, a novel method is presented for quantifying the steady movement of the blade tip's

7 chord line from its original position by using BTT data. The method assumes that the movement is of one given type of the three basic movements illustrated in Figs. 1a-c, although it is capable of

9 identifying translatory (axial or lean) from rotational (untwist) movement from the BTT data. Once

10 the amount of movement is determined, the actual measurement position of the probe relative to the

11 blade tip can be determined. The method depends only on the probe data and the chord stagger angle.

12 The method is divided into two main parts: (1) averaging the BTT displacements and monitoring the

13 change in this average along a range of rotational speed; (2) using the change in average to calculate

14 the amount of steady movement according to the geometrical relations between the BTT displacement and the expected movement. Although similar geometrical relations may already be used in previous studies, these have never been used to determine steady movements e.g. Battiato

17 [18] derived geometrical relations for quantifying out-of-plane vibration amplitudes. The current 18 method links geometrical relations to the BTT data in a unique approach in order to find the steady movements of the blade tip. The novel method depends only on data from a single probe in case of axial movement or blade lean, and from two probes placed at different axial positions and at the

21 same, or different, angular positions, in case of blade untwist. Such data are available in all BTT 22 measurement systems, and so there is no need for additional time or cost.

23 The novel method is validated in three ways: 
1 i. Using BTT data generated by a novel bladed disk ("blisk") simulator [13] developed by the authors.

3 ii. Using measurements from a test rig which is designed and built specifically for this purpose.

4 iii. Using real engine measurements from two different engines.

6 Validation tests (iii) test the reliability of the method for real applications since they are not conducted under the controlled conditions possible in the validation tests of (i) and (ii).

9 The preliminary research underpinning this method was first presented by the authors in a conference paper [13]. However, in contrast to the present paper, no experimental validation was presented in

11 [13]. Moreover, the rotational speed range used on the simulator in [13] did not include a resonance.

12 The inclusion of a critical (resonance) speed over the speed regime makes for a more interesting and 13 practical situation. The inclusion of resonance is more challenging since, in case of synchronous 14 vibration, the high rate of change in the amplitude of vibration at resonance may cause a conflict with the gradual change in average values of BTT displacements due to steady movements, unless 16 the method is able to distinguish between resonance and steady movements. Hence, the present 17 paper also shows how the data is processed to ensure correct steady movement calculation under conditions that include a resonance, and the preliminary validation tests (validation tests (i)) use the simulator to demonstrate the performance of the method over a speed regime that includes a critical 20 speed.

\section{BLADE TIP TIMING SYSTEM}

23 Blade tip timing systems consist of four main stages as shown in Fig. 3. The first stage of BTT 24 involves the use of instrumentation (probes and a data acquisition system) to measure and acquire the arrival (detection) times $t_{\mathrm{det}, j}$ of each given blade at a number of circumferentially distributed probes 
$1 j, j=1, \ldots, N_{\mathrm{Pr}}$. An additional probe, referred to as the once per revolution probe (OPR), is

2 typically used to determine the starting time of every revolution and keep track of the number of

3 revolutions completed, although some researchers have proposed to eliminate the OPR probe by

4 generating OPR signals from the blade arrival times, thus reducing costs [19].

5 The second stage of BTT is the processing of the collected data in order to calculate the tip 6 displacements of each blade at the angular positions of all probes during every revolution as follows

$$
d_{j}=\Omega R \Delta t_{j}
$$

7

\section{Fig. 3. HERE}

where $d_{j}$ is the instantaneous tip displacement in the plane of rotation ( $x$ - $y$ plane, see Fig. 3) as the blade passes probe $j, \Omega$ is the average angular speed ( $\mathrm{rad} / \mathrm{s})$ over a single revolution [19], $R$ is the radius of the blade tip measured from the centre of rotation, and $\Delta t_{j}$ is the difference between the blade's expected and the detected arrival times measured from the start of revolution

$$
\Delta t_{j}=t_{\mathrm{exp}, j}-t_{\mathrm{det}, j}
$$

The time $t_{\mathrm{exp}, j}$ is the expected time of arrival of the blade at probe $j$ if it rotated as a rigid body about the shaft axis. This can be calculated from the OPR probe as follows:

$$
t_{\mathrm{exp}, j}=t_{\mathrm{OPR}, n}+\left(\alpha_{j}-\beta_{i}\right) / \Omega
$$

where $t_{\mathrm{OPR}, n}$ is the start time of revolution no. $n, \alpha_{j}$ is the angular position (in $x-y$ plane) of the probe $j$ relative to the OPR probe (see Fig. 4), and $\beta_{i}$ is the angular position of the nominal sensing point on the undeformed blade relative to the OPR reference feature on the shaft, where both $\alpha_{j}, \beta_{i}$ are positive in the direction of rotation (see Fig. 4). 
$2 \Delta t_{j}$ in eq. (1) would therefore be zero in the absence of all of the following: blade vibration; angular

3 positional offsets of probes or blades; steady movement of the blade.

4 The third stage of BTT involves the analysis of the data in order to determine the vibration

5 parameters (amplitude, frequency, and phase). In this analysis, the instantaneous tip displacements $d_{j}$

6 of a given blade observed by probe no. $j$ (determined from eq. (1)) are assumed to follow the

7 following form in the time domain:

$$
\begin{aligned}
d_{j} & =P_{j}+\sum_{m=1}^{M}\left(a_{1 m} \sin \left(\omega_{m} t_{j}\right)+a_{2 m} \cos \left(\omega_{m} t_{j}\right)\right)+\text { noise } \\
& =P_{j}+\sum_{m=1}^{M}\left(a_{1 m} \sin \left(\mathrm{EO}_{m} \Omega t_{j}\right)+a_{2 m} \cos \left(\mathrm{EO}_{m} \Omega t_{j}\right)\right)+\text { noise }
\end{aligned}
$$

8 In eq. (4), $\omega_{m}=\mathrm{EO}_{m} \Omega, m=1 \ldots M$, is a generic frequency component, and $\mathrm{EO}_{m}$ is the associated

9 engine order (EO). The harmonic components can be either synchronous $\left(\mathrm{EO}_{m}\right.$ integer $)$ or non-

10 synchronous $\left(\mathrm{EO}_{m}\right.$ non-integer). $t_{j}$ in eq. (4) represents the sampling time according to the

11 assumptions made in the BTT algorithm used. $P_{j}$ is the steady offset resulting from the positional

12 errors of both probes and blades, in addition to the blade steady movements such as axial movements

13 and/or blade lean and blade untwist.

14 In the fourth and final stage of BTT, the estimated blade tip vibration is converted into equivalent 15 stress levels using an FE-based calibration model. These stress values are used to assign stress limits and improve the design of the bladed assembly. 
1 Figs. 5a,b show, for two different types of DC error, the tip displacement $d_{j}$ of a particular blade observed by a given probe $j$ over a large number of revolutions as the rotational speed is swept in order to traverse a blisk resonance.

Fig. 5(a,b). HERE

With reference to Fig. 5 and eq. (4), the steady offset $P_{j}$ in the expression for $d_{j}$ is the result of a DC error in the position of the probe relative to the blade arising from two sources:

a) Angular position errors (offsets) of the probes and/or the blades i.e. errors in $\alpha_{j}$ and $\beta_{i}$ in Fig. 4. From eq. (3), such speed-independent offsets $\Delta \alpha_{j}, \Delta \beta_{i}$ introduce errors in the expected time $t_{\text {exp, } j}$ and therefore in $\Delta t_{j}$ in eq. (2). However, due to division by $\Omega$ in eq. (3) and multiplication by $\Omega$ in eq. (1), the resulting error in $d_{j}$ is independent of speed $\Omega$. Hence, the contribution to the mean term $P_{j}$ arising purely from probe or blade positional offset is independent of speed, as seen in Fig. 5a. Probe or blade positional offset is outside the scope of this paper.

b) Steady (non-oscillatory) movement of the blade. As discussed in the Introduction these are caused by variations in operating conditions associated with a change in the rotational speed. As a consequence, $P_{j}$ would have a different value per revolution as the speed changes, as shown in Fig. 5b and explained in more detail below.

The basic types of steady movements of the blade tips were explained in the Introduction with reference to Figs. 1a-c. These movements have significant effect on the estimation of stress levels in blades from BTT measurements. Consider, for simplicity, the case of axial movement shown in Fig. 1a. Initially, point (1) has the same axial position of the probe $\left(z_{j}\right)$ represented by the dashed line. When its angular position intersects with the light beam of the probe, the time is recorded as the time of arrival (TOA) $t_{\mathrm{det}, j}$ of this blade at that probe during the current revolution, where $t_{\mathrm{det}, j}$ includes either an amount of delay or advance relative to the expected time of arrival $t_{\mathrm{exp}, j}$ according to the 
1 amplitude of vibration at point (1). If the blade moves axially with an amount $\Delta u^{\text {(off) }}$ as the speed

2 changes, a different point (point (2)) will coincide with the axial location of the probe $\left(z_{j}\right)$. Point (2)

3 will have an angular position of $\beta_{i}+\Delta \beta_{i}$ (Fig. 4), thus producing an effective shift in the expected

4 TOA equal to $\Delta t^{\text {(off) }}=\Delta \beta_{i} / \Omega$ (eq. (3)) as in the previous case of blade positional offset, except

5 that now $\Delta \beta_{i}$ will vary with speed. Thus, the resulting error in $d_{j}$ (eq. (1)) causes the mean in $d_{j}$ to

6 shift from its initial value $P_{j_{(1)}}$ (corresponding to position (1)) to a speed-dependent value $P_{j_{(2)}}$

7 corresponding to position (2), as was shown in Fig. 5b. The amplitude of vibration at point (2) is in

8 general different from that at point (1), which increases the uncertainty if point (1) is the nominal

9 sensing point used for correlating BTT measurements with FE predictions and stress conversions.

10 This is an extremely serious problem, as in some applications an amount of axial movement less than

$110.5 \mathrm{~mm}$ results in as much as $40 \%$ error in response amplitude [14]. Moreover, as already shown in

12 Figs. 2a,b, a shift in the sensing position can result in gross underestimation of the vibration 13 amplitude.

\section{DETERMINATION OF THE BTT MEASUREMENT POSITION}

The above-described effect of blade steady movement on the steady offset term in eq. (4) is exploited

to determine the amount of a given type of movement, thus enabling the location of the new sensing position of the probes relative to a given blade.

The first step of the new method is to extract from the BTT data the different values of $P_{j}$ as the

20 rotational speed is swept over a large number of revolutions. The change $\Delta P$ in $P_{j}$ over any sub-range

21 PQ within the complete range of revolutions of the test is then calculated as

$$
\Delta P=P_{j_{\mathrm{Q}}}-P_{j_{\mathrm{P}}}
$$


1 where $P_{j_{\mathrm{P}}}, P_{j_{\mathrm{Q}}}$ are the values of $P_{j}$ at the beginning and end respectively of the sub-range PQ, as

2 shown in Fig. 5b for the complete range of revolutions of the test. The last step is to calculate the magnitude of the movement corresponding to $\Delta P$ by applying an appropriate geometrical expression relating $\Delta P$ to the movement, according to its type. The sign of $\Delta P$ defines the direction of the movement. For example, if $\Delta P$ has a positive sign, this means that the steady movement causes the blade to arrive later, on average, than the expected time. So, the direction (sense) of a given type of movement can be specified by knowing the direction of rotation.

\subsection{The determination of the average values of BTT data}

This method is based on the data preparation method introduced by Russhard [7] who aimed to remove the steady offset from BTT data in order to enhance the identification of vibration by the BTT algorithms. With reference to eq. (4), the dataset comprising the blade tip displacements $d_{j}$ is first filtered to minimise non-synchronous components and noise using the Savitsky-Golay smoothing filters [20]. As the rotational speed is swept over a large number of revolutions, the speed-varying offset $P_{j}$ at any stage of the sweep can be determined by dividing the smoothed data $d_{j}$ into a large number of segments (buffers) each of length $N_{\text {buff }}$ points (where each point corresponds to a revolution) and taking a simple arithmetic average of each buffer or, in the resonance region, an interpolated average. With reference to eq. (4), the simple arithmetic average of each buffer extracts not only the data offset $P_{j}$, but also a large portion of the data associated with the synchronous (integral EO) components. The reason for this is that, for $\mathrm{EO}_{m}$ an integer, and gradual variations in rotational speed, the difference in $d_{j}$ from one revolution to the next is very small (there is no variation if the speed is constant). This is not a problem outside the resonance region where the vibration is low. However, in the region of resonance, the buffer averages will change rapidly according to change in vibration amplitude, which is in conflict with the gradual change in $P_{j}$. Hence, to make sure to capture $P_{j}$ only, a mask is placed on the resonance regions. If 
$1 \quad b_{k}$ is the simple arithmetic average of buffer no. $k$ and the masked region AB is bounded by buffer

2 nos. $k_{\mathrm{A}}$ and $k_{\mathrm{B}}$, then the value of $P_{j}$ for buffer no. $k$ is calculated as follows:

3

4

5

6
- for $k<k_{\mathrm{A}}, k>k_{\mathrm{B}}$ (buffers outside $\mathrm{AB}$ ) - $P_{j}$ is calculated by the simple arithmetic average of the $d_{j}$ data in the buffer.

- for $k_{\mathrm{A}}<k<k_{\mathrm{B}}$ (buffers inside $\mathrm{AB}$ ) - $P_{j}$ is calculated by linear interpolation between $b_{k_{\mathrm{A}}}$ and $b_{k_{\mathrm{B}}}$.

The above method is explained in Fig. 6, which plots blade displacement data $d_{j}$ containing a single synchronous harmonic component with $\mathrm{EO}$ of $2\left(M=1, \mathrm{EO}_{1}=2\right.$ in eq. (4)) over a large number of revolutions as the rotational speed traverses a resonance (this dataset is used in one of the validation tests in section 6).

\section{Fig. 6. HERE}

The above method of calculating $P_{j}$ is justified since: (i) the vibration amplitude outside the resonance region is low; (ii) the variation in the non-oscillatory (steady) movements is slow, so the use of averages of buffers each covering a number of revolutions is valid, as is the interpolation across resonances.

The resonance regions to be masked are identified by examining the coherence of the displacement values based on revolution-by-revolution technique using the following cross correlation factor $\left(X_{r}\right)$ by Carrington [21].

$$
X_{r}=\frac{2 \sum_{j=1}^{N_{\operatorname{Pr}}}\left({ }^{n} d_{j}\right)\left({ }^{n+1} d_{j}\right)}{\sum_{j=1}^{N_{\operatorname{Pr}}}\left({ }^{n} d_{j}\right)^{2}+\sum_{j=1}^{N_{\operatorname{Pr}}}\left({ }^{n+1} d_{j}\right)^{2}}
$$


1 where ${ }^{n} d_{j}$ is the displacement of a given blade observed by probe no. $j$ during revolution no. $n$.

2 According to [7], the value of $X_{r}^{2}$ should be close to 0.99 based on experiments for amplitudes in the

3 resonance region of at least $0.1 \mathrm{~mm}$. Based on the authors' experiments using the simulator, the

4 value of $X_{r}$ remains constant until the beginning of resonance region and then reduces to a certain

5 level, before increasing again, going back to the original value at the end of resonance. Also, it was

6 reported [7] that the minimum number of probes required for the $X_{r}$ formula (eq. 6a) to be valid

7 depends on the number of excitation frequencies $M$ and can be calculated as

$$
\left.N_{P}\right|_{\min }=2 M+2
$$

8 Note that, if the number of probes is less than $\left.N_{P}\right|_{\min }, X_{r}$ would always give a value of 1 [7].

\subsection{Geometrical relation for axial movement}

Fig. 7 shows a blade tip that has moved axially. The blade has a chord line angle $\psi$ relative to a horizontal line parallel to the axis of rotation (z-axis). The magnified triangle represents the relation between $\Delta P$, the amount of axial movement $A_{1}$, and the shift $A_{2}$ in the sensing position along the chord line, which is given by the following expressions:

$$
\begin{aligned}
& A_{1}=\Delta P / \tan \psi \\
& A_{2}=\Delta P / \sin \psi
\end{aligned}
$$

\subsection{Geometrical relation for lean}

17 Fig. 8 shows a blade tip that is subjected to lean. The magnified triangle represents the relationship between $\Delta P$, the amount of lean $A_{1}$, and the shift $A_{2}$ in the sensing position along the chord line, which can be expressed as follows: 


$$
\begin{aligned}
& A_{1}=\Delta P \cos \psi \\
& A_{2}=\Delta P \sin \psi
\end{aligned}
$$

5 The identification of the untwist movement includes the determination of two parameters, the untwist angle $\emptyset$, and the position of the axis of twist $l$ measured from the point of intersection between the axial position of a probe and the non-deformed blade, as shown in Fig. 9. Therefore, two probes at different axial positions are required. However, they can be placed at any two angular positions,

9 which can be either different or the same. The following relation can be obtained using the triangles $10\left(a-b-b^{\prime}\right)$ and $\left(a-c-c^{\prime}\right)$ in Fig. 9

$$
\tan (\psi+\varnothing)=\frac{\Delta P_{2}+\left(l \sin \psi-\Delta z_{\mathrm{p}} \tan \psi\right)}{l \cos \psi-\Delta z_{\mathrm{p}}}=\frac{\Delta P_{1}+l \sin \psi}{l \cos \psi}
$$

11 where $\Delta P_{1}$ and $\Delta P_{2}$ are the changes in the steady offsets of the data from the left and right probes

12 respectively, and $\Delta z_{\mathrm{p}}$ is the axial distance between the two probes. Rearranging eq. (9) results in the

13 following expression

$$
l\left[\Delta P_{2} \cos \psi-\Delta P_{1} \cos \psi\right]+\Delta P_{1} \Delta z_{p}=0
$$

14 from which $l$ can be calculated as follows

$$
l=\Delta P_{1}\left(\Delta z_{\mathrm{p}} / \cos \psi\right) /\left(\Delta P_{1}-\Delta P_{2}\right)
$$


1 Knowing the value of $l$ and by using eq. (9), the untwist angle $\varnothing$ can be calculated using the

2 following formula

$$
\emptyset=\tan ^{-1}\left[\left(\Delta P_{1}+l \sin \psi\right) / l \cos \psi\right]-\psi
$$

3 Finally, the distances $\overline{a c^{\prime}}, \overline{a b^{\prime}}$ of the new sensing points on the blade for the two probes $\mathrm{P}_{1}, \mathrm{P}_{2}$ 4 respectively, measured along the chord from the axis of twist, are calculated as follows

$$
\begin{aligned}
& \overline{a c^{\prime}}=l \cos \psi / \cos (\psi+\emptyset) \\
& \overline{a b^{\prime}}=\left(l \cos \psi-\Delta z_{\mathrm{p}}\right) / \cos (\psi+\emptyset)
\end{aligned}
$$

5 The use of two probes at different axial positions may also be useful for the differentiation between

6 the untwist movement and both axial and lean movements. If $\Delta P_{1}=\Delta P_{2}$, then the movement is either 7 axial, lean, or a combination of both. If $\Delta P_{1} \neq \Delta P_{2}$ then the movement is purely untwist.

\section{BTT SIMULATOR}

9 The evaluation of the new BTT methods is usually carried out using data from simulators instead of real measurements, especially during the early stages of evaluation. This can save a huge amount of cost and time, especially since simulators allow tests to be conducted under a variety of controlled conditions. The previous BTT simulators have been based on simple mathematical models (discrete multi-degree-of-freedom spring-mass systems) to study the dynamic behaviour of bladed systems under controlled conditions [22,23]. These models had a number of limitations as follows [13]:

- Blade deflection is restricted to a single degree of freedom;

- Elastic coupling between non-adjacent blades is neglected;

- Rigid supports are used for the intermediate connection between the blades and the disk;

- A variety of excitations and/or steady movements cannot be applied to such systems. 
1 On the other hand, as observed in [13], the data generated by most commercially available BTT

2 simulators are not derived from a simulation of the actual mechanics of a blisk. Hence, the dynamics

3 of currently available or published simulated systems are not likely to correspond to those of a real

4 blisk, thus compromising the results of evaluations of BTT algorithms. Indeed, it has been observed

5 [7] that algorithms may work very well with data from simplistic simulators but fail to achieve

6 sufficient accuracy with real data. It is also noted that FE-based simulators used in previous works

7 (Khayrton [24], Diamond [25]) were limited to single mode resonance [26].

8 For the above reasons, a simulator that is based on a realistic (FE-derived) multi-modal model of the

9 blisk, and which can accurately capture the complexity of the blisk design (and related dynamics)

10 while retaining computational efficiency, was developed by the authors. An overview of this

11 simulator was presented in [13].

12 If $\mathbf{u}(t)$ denotes the $3 K \times 1$ vector containing the instantaneous absolute coordinates of the nodes $\mathrm{P}_{k}$

$13(k=1 \ldots K)$ on the tip of a given blade, the simulator computes $\mathbf{u}(t)$ as follows

$$
\mathbf{u}(t)=\mathbf{u}^{(\mathrm{rgd})}(t)+\Delta \mathbf{u}^{(\mathrm{def})}(t)+\Delta \mathbf{u}^{(\mathrm{off})}(t)
$$

where:

- $\mathbf{u}^{(\mathrm{rgd})}(t)$ represents the blade tip positions due to rotation of the blisk as a rigid body (in the absence of deformation and any applied shifting)

- $\Delta \mathbf{u}^{(\text {def) }}(t)$ represents the dynamic (deformation) response to the applied dynamic excitation forces.

- $\Delta \mathbf{u}^{(\text {off }}(t)$ represents a steady shift prescribed to the blade tip that can vary with time. This component is used in the preliminary validation tests of section 6.1 to simulate the steady movements to be determined by the BTT method from the BTT data generated by the simulator: 

axial movement; blade lean; blade untwist. Each type of movement is applied in a separate simulation, but it should be noted that the simulator is capable of superimpose all movements.

3 The term $\Delta \mathbf{u}^{(\mathrm{def})}(t)$ represents the oscillatory part of eq. (14). Since it is superimposed on the rigid 4 body rotation and the prescribed steady shift in eq. (14), $\Delta \mathbf{u}^{(\mathrm{def})}(t)$ is determined by considering the 5 dynamic response of the non-rotating blisk in a fixed reference angular position. The elements of

$6 \Delta \mathbf{u}^{(\mathrm{def})}(t)$ are calculated from a transformation to modal space using as basis functions the first $H$ 7 natural undamped modes of vibration of the non-rotating blisk in the reference angular position:

$$
\Delta \mathbf{u}^{(\mathrm{def})}(t)=\mathbf{H}_{\mathrm{P}} \mathbf{q}(t)
$$

8 where $\mathbf{q}(t)$ is the $H \times 1$ vector of modal co-ordinates that are governed by the modal equations of 9 motion:

$$
\ddot{\mathbf{q}}(t)+\operatorname{diag}\left(\left[\cdots 2 \zeta_{r} \varpi_{r} \cdots\right]\right) \dot{\mathbf{q}}(t)+\operatorname{diag}\left(\left[\cdots \varpi_{r}^{2} \cdots\right]\right) \mathbf{q}(t)=\mathbf{H}_{\mathbf{f}}^{\mathrm{T}} \mathbf{f}(t)
$$

11 In eqs. (15) and (16):

- $\mathbf{H}_{\mathrm{P}}, \mathbf{H}_{\mathbf{f}}$ are modal transformation matrices whose $H$ columns are mass-normalised eigenvectors corresponding to the natural circular frequencies $\varpi_{r}(r=1, \ldots, H)$ evaluated at the appropriate degrees of freedom. The modal parameters in the reference angular position are determined by a one-off FE modal analysis of a given blisk that is fixed (fully constrained) at its hub.

- $\mathbf{f}(t)$ is the $3 N \times 1$ vector of dynamic excitation forces applied to the blisk (frozen at its reference angular position) at nodes $Q_{n}(n=1 \ldots N)$ in the directions of the Cartesian degrees of freedom there: 


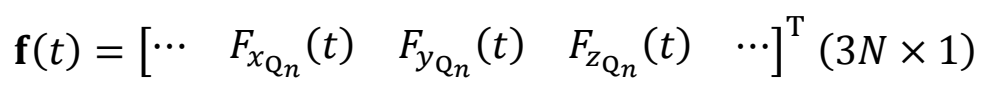

2 For the validation of the method developed in the current study, a travelling wave excitation force is 3 applied in a direction perpendicular to the plane of rotation, and at nodes $Q_{n}(n=1 \ldots . N)$ at an edge

4 of every blade tip. For an angular velocity sweep of duration $T_{\mathrm{s}}$, the excitation force in this paper is 5 chosen to excite one particular harmonic component of the assumed displacement response in eq. (4), 6 and is therefore a chirp signal whose frequency is a fixed ratio $\mathrm{EO}_{m}$ of the time-varying angular 7 speed, as described by the following formula [23]

$$
F_{z_{\mathrm{Q}_{n}}}(t)=A_{z_{\mathrm{Q}_{n}}}^{(m)} \sin \left[\mathrm{EO}_{m}\left(\Omega_{\mathrm{i}} t+\left(\frac{\Omega_{\mathrm{f}}-\Omega_{\mathrm{i}}}{2 T_{\mathrm{s}}}\right) t^{2}\right)+\varphi_{z_{\mathrm{Q}_{n}}}^{(m)}\right]
$$

8 where $A_{z_{Q_{n}}}^{(m)}$ and $\varphi_{z_{Q_{n}}}^{(m)}$ are the force amplitude and phase respectively corresponding to the frequency 9 component in eq. (4) with engine order $\mathrm{EO}_{m}$, and $\Omega_{\mathrm{i}}, \Omega_{\mathrm{f}}$ are the initial and final angular velocities $10(\mathrm{rad} / \mathrm{s})$ respectively. The phase angles are set according to the following formula [27] in order to 11 obtain the travelling wave model of blade excitation

$$
\varphi_{z_{\mathrm{Q}_{n}}}^{(m)}=2 \pi i N_{\mathrm{ND}} / N_{\mathrm{b}} \quad i=0,1, \ldots,\left(N_{\mathrm{b}}-1\right)
$$

12 where $N_{\mathrm{ND}}$ is the number of nodal diameters, $i$ is the number of the blade to which node $\mathrm{Q}_{n}$ belongs, and $N_{\mathrm{b}}$ is the total number of blades.

14 The simulator is implemented in Matlab/Simulink and solves eq. (16) using a numerical integration 15 routine with automatic time-step control. At each time step it combines the computed $\Delta \mathbf{u}^{(\mathrm{def})}(t)$ 16 (eq. (15)) with $\mathbf{u}^{(\mathrm{rgd})}(t)$ and $\Delta \mathbf{u}^{(\mathrm{off})}(t)$ to determine $\mathbf{u}(t)$ as per eq. (14). For a given configuration 17 of probes, $t_{\text {exp }, j}$ is determined from $\mathbf{u}^{(\mathrm{rgd})}(t)$ by locating the passing time of the node on the blade 
tip that coincides with the angular and axial position of probe no. $j$. The actual arrival time $t_{\mathrm{det}, j}$ is

2 determined in the same way but using $\mathbf{u}(t)$ instead of $\mathbf{u}^{(\mathrm{rgd})}(t)$. The blade tip displacements $d_{j}$ are

3 then determined as per eqs. (1) and (2) and corrupted with Gaussian white noise of prescribed noise-

4 to-signal ratio (NSR) to simulate AC measurement noise.

5 The present version of the simulator does not consider rotational effects such as gyroscopic, Coriolis,

6 and centrifugal stiffening effects. This is justified for the present simulations through the use of a

7 lightweight blisk.

\section{VALIDATION OF THE METHOD}

The ability of the method developed in section 5 to determine the amount of steady movement of a blade tip from BTT data was validated using BTT datasets from three different types of sources:

- Simulated data developed by a BTT simulator - this will be used to test the method on all

\subsection{Validation using simulator for axial movement, lean, and untwist}

20 A FE model of the blisk shown in Fig. 10 was established using SolidWorks for the construction of

21 its geometry, and then exported to ANSYS 15.0 for building the model and performing the required modal analysis. The modal and the geometrical properties of the model were used as the inputs to the 
1 simulator described in section 5, in addition to the information on the rotational speed, probe

2 configuration, excitation forces, and the prescribed steady movement of the blade tip. The simulator

3 generated the BTT displacements of a given blade as seen by all the probes within the time range of

4 the angular velocity sweep, and the data were corrupted by noise as described in section 5 . The data

5 were then processed for the steady offset as per method described in section 4.1. Finally, the amount

6 of movement was calculated as per sections 4.2 to 4.4 and the result compared with the prescribed

7 value.

Fig. 10. HERE

The material of the blisk was aluminium $\left(2750 \mathrm{~kg} / \mathrm{m}^{3}\right.$ density and $72 \mathrm{GPa}$ elasticity modulus $)$. It consisted of a disk of thickness $16 \mathrm{~mm}$ with inner diameter of $50 \mathrm{~mm}$ and an outer diameter of 180 $\mathrm{mm}$ at which were integrally attached 40 inclined flat blades. Each blade was $90 \mathrm{~mm}$ long, $6 \mathrm{~mm}$ thickness, and $60^{\circ}$ pitch (angle between chord line and plane of disk). The FE model had 1791261 degrees-of-freedom (DOFs), and the 10-noded solid element SOLID187 was used for meshing. The mesh was highly refined at the tip of some of the blades as shown in Fig. 10 in order to minimize the distance between the different nodes along the width of the blade tip. This improved the accuracy of the simulator to detect the time of arrival (TOA) (ideally, the tip surface would have an infinity of nodes). The disk centre hole (the hub) was completely restrained during the modal analysis of the static blisk in the reference angular position, as described in section 5 . The first five natural frequencies of the blisk are listed in Table 1, and their corresponding mode shapes are shown in Fig. 
Table 1. Natural frequencies of the blisk.

\begin{tabular}{llllll}
\hline Mode No. & 1 & 2 & 3 & 4 & 5 \\
\hline Type & bending & bending & axial & bending & bending \\
\hline $\begin{array}{lllll}\text { Frequency }(\mathrm{Hz}) \\
\begin{array}{l}\text { Number of nodal } \\
\text { diameters }\end{array}\end{array}$ & 340.8 & 340.8 & 361.5 & 392.9 & 393.0 \\
\hline
\end{tabular}

2

3 With regard to eq. (18), the dynamic excitation force used in the simulator had an amplitude of $A_{z_{Q_{n}}}^{(m)}$

4 of $100 \mathrm{~N}$, engine order $\mathrm{EO}_{m}=2$, and the phase angles $\varphi_{z_{\mathrm{Q}_{n}}}^{(m)}$ in eq. (19) were selected based on a

5 single nodal diameter $\left(N_{\mathrm{ND}}=1\right)$ in order to excite the first bending mode (Figures 11(a,b)). The

6 sweeping rotational speed increased from zero at $t=0$ to $350 \mathrm{rev} / \mathrm{s}$ at the end of simulation $(t=$

$72.1 \mathrm{~s}$ ), resulting in an excitation frequency range of $0-700 \mathrm{~Hz}$. The amount of noise added to the

8 simulated BTT displacement data was $10 \%$ noise-to-signal ratio (NSR).

9

10 In accordance with the method in section 4, only two probes were used to determine the steady

11 movements, their angular positions being $5^{\circ}$ and $10^{\circ}$ anticlockwise relative to positive direction of

12 the $x$ axis (Fig. 3), and their axial positions located at $z=5 \mathrm{~mm}$ and $z=-5 \mathrm{~mm}$. However,

13 additional probes were used and placed at different angular positions $\left(10^{\circ}, 60^{\circ}, 110^{\circ}\right.$, and $\left.160^{\circ}\right)$ for

14 the purpose of identifying and masking the resonance areas in accordance with the method of section

15 4.1. This was because the angular spacing between the two probes used to determine the steady

16 movements was small, thus producing little phase difference between the signals measured at these

17 two probes, and the identification of resonance via the correlation eq. (6a) requires significant

18 variation between the data from different probes.

Fig. 11. HERE 
1 Three different simulations were implemented which included the following total movements over

2 the angular velocity sweep:

- a prescribed axial movement of $1 \mathrm{~mm}$;

- a prescribed lean movement of $1 \mathrm{~mm}$;

- a prescribed untwist angle of $5^{\circ}$.

7 The BTT displacements of a blade tip from the three simulations including the added noise are

8 shown in Figs. 12a-c. The steady offset $P_{j}$ was then extracted for each situation according to the 9 method described in section 4.1, where the number of points (revolutions) in each buffer was $N_{\text {buff }}=10$, and the corresponding results are shown in Figs. 13a-c. The amounts of movement were 11 then calculated using eqs (7), (8), and (12), and compared to the prescribed values. As shown in 12 Table 2, the percentage errors of all cases were found to be negligible (less than $3 \%$ ). The highest 13 error $(-2.8 \%)$ was for the untwist movement - this was expected due to the reliance on data from two 14 probes, which increases the probability of error.

Fig. 13. HERE

Table 2. Comparison between prescribed and calculated amounts of movement (excitation force 21 amplitude of $100 \mathrm{~N})$.

\begin{tabular}{llll}
\hline Type of movement & Axial $(\mathrm{mm})$ & Lean $(\mathrm{mm})$ & Untwist (degrees) \\
\hline Prescribed & 1 & 1 & 5 \\
\hline Measured & 0.982 & 0.993 & 4.86 \\
\hline$\%$ Error & -1.8 & -0.7 & -2.8 \\
\hline
\end{tabular}


1 Another three simulations are carried out using higher force amplitude (300 N), in order to check its

2 effect on the results. Table 3 shows the results of the new simulations, where is seen that the errors

3 are almost the same as before in case of lean movement, while they are reduced in case of both axial,

4 and untwist.

6 Table 3. Comparison between prescribed and calculated amounts of movement (excitation force amplitude of $300 \mathrm{~N}$ ).

\begin{tabular}{llll}
\hline Type of movement & Axial $(\mathrm{mm})$ & Lean $(\mathrm{mm})$ & Untwist (degrees) \\
\hline Prescribed & 1 & 1 & 5 \\
\hline Measured & 0.99 & 0.992 & 4.88 \\
\hline$\%$ Error & -1.0 & -0.8 & -2.4 \\
\hline
\end{tabular}

7

8 The errors in Tables 2 and 3 probably arise from the following sources:

- $\quad$ The filtering and averaging processes in section 4.1.

- The finite distance between adjacent FE nodes on the blade tip.

- A tiny part of steady movement might be missed from the BTT output data since the first blade detection happened just after the movement started and the last detection happened just before the movement stopped. This is, most probably, the reason for the negative sign of the error in all results in Tables 2 and 3.

The last source of error can be avoided by starting the movement after at least 2 or 3 revolutions from the beginning of simulation, and stopping it at least 2 or 3 revolutions before the end. It should also be noted that the second source of error is not an issue with real BTT data.

In general, the above results prove the validity of the new method since it has been shown to be capable of quantifying all three types of steady blade tip movement (axial, lean, or untwist) with a 
1 high level of accuracy using BTT data generated by a realistic blisk simulator under controlled 2 conditions.

\subsection{Experimental validation on test rig for axial movement}

5 Fig. 14 shows the test rig, which is installed at the testing facilities of Rotadata Ltd. in Derby, UK.

6 The rig includes a new feature of adding steady axial movement to the measurements by moving the casing (including the probes) axially by means of a screw driven by a stepper motor. A blisk with 18

8 integrated inclined flat blades is used and the pitch angle (angle between chord line and plane of

9 rotation) of each blade is $42^{\circ}$. Seven probes are fitted into the casing at the same axial positions, with

10 the angular positions shown in Table 4. Two tests were carried out at the same constant speed (about $112420 \mathrm{rpm})$, each including the same magnitude of axial movement $(3 \mathrm{~mm})$, but in opposite

12 directions. Notice that, unlike the other validation tests in this paper, these tests were conducted 13 under constant speed of the blisk. However, this is not an issue since the axial movement in this rig 14 is controlled by the stepper motor independently from the rotational speed of the blisk.

Fig. 14. HERE

Table 4. Test rig probes angles (measured anticlockwise from positive $x$-axis, Fig. 3).

\begin{tabular}{llllllll}
\hline Probe No. & 1 & 2 & 3 & 4 & 5 & 6 & 7 \\
\hline Angle (degrees) & 0 & 40.52 & 57.3 & 79.66 & 131.08 & 146.72 & 174.12 \\
\hline
\end{tabular}

20

$21 \quad$ 6.2.1 First test 
1 With reference to the first test (prescribed axial movement of $+3 \mathrm{~mm}$ ), Figs. 15a-c show the BTT

2 displacements of one blade at all probes during the total number of revolutions of the test (19924

3 revolutions). The results appear to show that the peak-to-peak displacement was about $2 \mathrm{~mm}$ even

4 though no excitation applied during the test. By studying the data in the frequency domain it was

5 found that this high response had a low frequency, which is typical of noise interference from the

6 OPR signal and the casing holding the probes. According to the industrial collaborator, this effect is

7 often encountered in real testing and there is currently no method that can remove it without also

8 removing the data shift due to steady movements. However, a measurement method that does not

9 need an OPR probe, such as the one proposed in [19], can be tried in future experiments. It is also

10 clear from visual observations that the measurements from both the second and third probes (Fig.

11 15c) were not consistent with those from the other probes (Figs. 15a,b), and so they were excluded

12 from the data analysis. This had no effect on the current method since there were another 5 probes

which gave consistent data, and the method only needs two probes for extracting the amount of movement. The data were filtered using Savitsky-Golay smoothing filters in order to remove noise and averaged as per method in section 4.1 and the steady offset $P_{j}$ calculated as shown in Fig. 16 for the data of probe number 1 (each data buffer containing $N_{\text {buff }}=400$ points, where each point corresponds to a revolution). It is noted that no masking for resonance was needed in this case. The variation in axial position of the blade with the number of revolutions (from one buffer to the next) was calculated for each probe by applying eq. (7) and using the change $\Delta P$ in the steady offset $P_{j}$ from one buffer to the next, as shown in Fig. 17. The total calculated value of axial movement (covering the complete range of revolutions) is not precisely the same for all probes. The mean of these overall values was computed as $2.9 \mathrm{~mm}$, which was $3.3 \%$ less than the actual value of the prescribed movement $(3 \mathrm{~mm})$. The low error proves the accuracy of the method despite the high level of noise. 


\section{$4 \quad \underline{6.2 .2 \text { Second test }}$}

5 With reference to the second test (prescribed axial movement of $-3 \mathrm{~mm}$ ), Figs. 18a-c show the BTT 6 displacements of one blade at all probes during the total number of revolutions of the test (18545 7 revolutions). Once again, the data from the second and third probes (Fig. 18c) were found to be

8 inconsistent with those from the other probes (Figs. 18a,b) and were therefore excluded. Similarly to

9 the first test, the offset $P_{j}$ was calculated as shown in Fig. 19 for the data of probe number 1 (each 10 data buffer containing $N_{\text {buff }}=400$ points, where each point corresponds to a revolution), and the 11 variation in the axial position determined from the data of each probe as shown in Fig. 20. The mean 12 of the overall values of axial movement determined from all used probes was calculated to be -2.8 $13 \mathrm{~mm}$, which was $6.7 \%$ different from the actual prescribed value $(-3 \mathrm{~mm})$.

14 The above experimental results prove the validity of the developed method for the determination of 15 the magnitude and direction (sense) of the steady axial movement of a rotating blisk using BTT data measured from a laboratory test rig under controlled conditions. The facility to impose a controlled amount of blade lean or blade untwist in laboratory conditions would require a test rig that is considerably more complex in design which was beyond the resources available for this research 19 project.

Fig. 18. HERE

Fig. 19. HERE 
1 In sections 6.1 and 6.2 the new method was validated using simulated data and experimental data

2 respectively, both under controlled conditions. The tests in this section assess the reliability of the

3 method when applied to real applications where measurements are obtained under operational

4 conditions. Two sets of data from two different applications and conditions are provided for this

5 study. The industrial provider of these datasets placed confidentiality restrictions on the publication

6 of some of the details of the engine tests.

\section{$7 \quad$ 6.3.1 First engine test}

8 Table 5. Probe positions used in first engine test.

\begin{tabular}{llllll}
\hline Probe No. & 1 & 2 & 3 & 4 & 5 \\
\hline $\begin{array}{l}\text { Angle (degrees) (anti- } \\
\text { clockwise from } x \text {-axis, Fig. 3) }\end{array}$ & 17.1 & 21.0 & 26.0 & 31.0 & 36.0 \\
\hline Axial position (mm) & 0 & 30.0 & 70.0 & 70.0 & 150.0 \\
\hline
\end{tabular}

9

The first system included 24 blades. Each blade had a pitch angle of $69^{\circ}$ and tip radius of about $1.5 \mathrm{~m}$ measured from the centre of rotation. Five probes were used at different angular and axial positions as shown in Table 5. The change in the rotational speed during the BTT measurements was as shown in Fig. 21. The total time of measurements is $80 \mathrm{~s}$, and the total number of revolutions is 1752 . According to the provider of these measurements, the blades were subjected to untwist. The untwist angle of the first blade tip at the maximum speed was known to be $2.8^{\circ}$, according to independent estimates made by the provider of the data. In addition to observing untwist, the industrial provider also observed a $10 \mathrm{~mm}$ steady movement at the blade tips in a direction normal to the plane of rotation (i.e. the axial direction). However, as discussed below, the analysis of this paper indicated that such axial movement was a consequence of the untwist rather than the independent component of axial movement defined in Fig. 1a (where each point on the chord has the same axial 
displacement). It is therefore referred to as "equivalent axial movement due to untwist" (with each

2 point on the chord undergoing a different axial displacement).

Fig. 21. HERE

The BTT displacements of the first blade at all probes are shown in Fig. 22. The measurements from each probe were processed (filtered and averaged), and the variation in steady offset $P_{j}$ within the time of measurement was then extracted using the averaging method of section 4.1, as shown in Fig. 23 for the data of probe (1) (each data buffer contained $N_{\text {buff }}=100$ points). The calculations of the untwist angle by the novel method presented in this paper require data from two probes at different axial positions. Therefore there were nine combinations of data that could be used from the available five probes. The variation in the untwist angle of the first blade angle was calculated using eq. (12) and is shown in Fig. 24. The estimates for untwist angle at the maximum speed, computed using the nine different combinations of data, are close but not precisely the same, of course. The mean value for untwist angle at maximum speed was computed as $2.43^{\circ}$, which was around $13.2 \%$ different from the value given by the industrial collaborator $\left(2.8^{\circ}\right)$. Note that, the given value was determined using one of the validated methods mentioned in the literature which either require the use of additional equipment, or are limited to specific types of blades.

The variation with time of the equivalent axial movement of the first blade tip due to the blade untwist was calculated by eq. (7) and using the data of each probe as shown in Fig. 25. The maximum value of axial movement was found to be $11.8 \mathrm{~mm}$. This was about $18 \%$ different from the approximate value observed by the industrial provider $(10 \mathrm{~mm})$. The data from the other probes gave different values of axial movement both in terms of magnitude, and direction, due to their different positions relative to the axis of twist.

Fig. 22. HERE 


\subsubsection{Second engine test}

6 The second set of data was for a system of 20 blades, each blade having a pitch angle of $66.7^{\circ}$. Two
Fig. 23. HERE

Fig. 24. HERE

Fig. 25. HERE probes were used at $89.4^{\circ}$ and $101.7^{\circ}$ angular positions (measured anticlockwise from $x$-axis, Fig. 3), with axial positions of 0 and $40 \mathrm{~mm}$ respectively. The total time of the measurements was $570 \mathrm{sec}$, covering 26596 revolutions. The change of speed along the time of measurements is shown in Fig. 26. The blades were subjected to untwist, and the change in blade tip angle at the end of the measurements, relative to the start, was known to be $-0.7^{\circ}$ according to the independent estimates made by the provider of the data. The BTT displacements of the first blade at the two probes are shown in Fig. 27. The data were processed (filtered and averaged) and averaged for the steady offset according to the method of section 4.1, as shown in Fig. 28 and Fig. 29 for the first and second probes respectively. The number of points used in each buffer was $N_{\text {buff }}=300$. Two resonance regions were observed at about $40 \mathrm{~Hz}$ and $170 \mathrm{~Hz}$. However, it was not possible to confirm this using the correlation factor of eq. (6a) because more probes were required at different angular positions intervals, as per eq. (6b). The change in untwist angle was calculated by eq. (12) using data from the only available probe-pair combination, as shown in Fig. 30. Finally, the change in untwist angle at the end of measurements relative to the start was computed as $-0.78^{\circ}$, which was $11.4 \%$ different from the given value $\left(-0.7^{\circ}\right)$. This also proves that the insufficient information for the accurate specification of resonance regions did not affect the results since the magnitude of the changes in the average values due to untwist were relatively higher than the amplitude of resonance. In summary, the method once again proved its validity using real measurements, within reasonable error margins. 
Fig. 27. HERE

Fig. 28. HERE

Fig. 29. HERE

Fig. 30. HERE

\section{CONCLUSIONS}

A novel method has been developed for the determination of the amount of steady (non-oscillatory) displacement of a blade tip. This enables the identification of the correct sensing position of each BTT probe relative to the blade tip, thus avoiding potentially serious errors in estimating blade vibration amplitudes and associated stress levels. The types of movement considered were: axial movement; blade lean; and blade untwist. The method was based on using the BTT displacement measurements from one or two probes, extracting the variation in the steady offset along a number of revolutions for each probe, and then calculating the amount of steady movement using a geometrical relation corresponding to the type of movement. The method has been validated using BTT datasets from three different types of sources: (i) a novel simulator that can generate BTT data for a realistic model of a blisk based on a FE-derived modal model, and allowing the application of any of the three types of steady movement; (ii) BTT measurements from a purpose-built test rig that allows the application of a controlled amount of axial movement; (iii) two engine tests providing real engine data including the effect of blade untwist. The simulator tests (i) validated the method for the calculation of all three types of movement, since the errors were always below 3\%, and lower for higher excitation force amplitude. The maximum error was found to be that for untwist, which was expected because it required data from two probes, thus increasing the probability of error. For the validation tests (ii) using the test rig, the error in axial movement did not exceed $7 \%$, and the method was able to correctly identify the direction (sense) of movement in the two cases. For the two real engine data tests (iii), the untwist angle calculated by the novel method did not deviate by more than $13.2 \%$ from the value divulged by the provider of the engine test data after the calculations were 
performed. All these results provide strong evidence that the novel method presented in this paper is capable of achieving satisfactory levels of accuracy in real engine applications, eventually allowing it to be used in the field with a reasonably high level of confidence. Further experimental validation tests are required, particularly in the case of lean (which was only tested using the simulator in this paper). Also, the method needs to be developed to deal with simultaneous steady movements of the blades (i.e. combinations of axial shift / lean / untwist). Moreover, the applicability of the method to different types of probes, other than optical probes, also needs to be examined.

\section{ACKNOWLEDGMENT}

We would like to thank the Egyptian Ministry of Higher Education, and the British Council in Cairo

for funding this project. We also wish to express our sincere thanks to Rotadata Ltd, Derby, UK, for its support, including access to instrumentation and facilities.

\section{REFERENCES}

[1] S. Heath, A Study of Tip-Timing Techniques for the Determination of Bladed Disk Vibration Characteristics, University of London, 1996.

[2] S. Heath, M. Imregun, An Improved Single-Parameter Tip-Timing Method For Turbomachinery Blade Vibration Measurement Using Optical Laser Probes, International Journal of Mechanical Sciences. 38 (1996) 1047-1058.

S. Heath, A New Technique For Identifying Synchronous Resonances Using Tip-Timing, Journal of Engineering for Gas Turbines and Power-Transactions of the ASME. 122 (2000) 219-225.

[4] I.B. Carrington, J.R. Wright, J.E. Cooper, G. Dimitriadis, A comparison of blade tip timing data analysis methods, Proceedings of the Institution of Mechanical Engineers, Part G: Journal of Aerospace Engineering. 215 (2001) 301-312.

[5] J. Gallego-Garrido, G. Dimitriadis, J.R. Wright, A Class of Methods for the Analysis of Blade Tip Timing Data from Bladed Assemblies Undergoing Simultaneous Resonances-Part I: Theoretical Development, International Journal of Rotating Machinery. (2007) 1-11.

[6] J. Gallego-Garrido, G. Dimitriadis, I.B. Carrington, J.R. Wright, A Class of Methods for the Analysis of Blade Tip Timing Data from Bladed Assemblies Undergoing Simultaneous 
Resonances-Part II: Experimental Validation, International Journal of Rotating Machinery. (2007) 1-10.

[7] P. Russhard, Development of a Blade Tip Timing Based Engine Health Monitoring System, The University of Manchester, 2010.

[8] G. Rigosi, G. Battiato, T.M. Berruti, Synchronous vibration parameters identification by tip timing measurements, Mechanics Research Communications. 79 (2016) 7-14.

[9] J. Lin, Z. Hu, Z.S. Chen, Y.M. Yang, H.L. Xu, Sparse reconstruction of blade tip-Timing signals for multi-mode blade vibration monitoring, Mechanical Systems and Signal Processing. 81 (2016) 250-258.

[10] Y.-J.Y. and A. von F. Kyu-Kang Joung, Suk-Chul Kang, Ki-Seok Paeng, No-Gill Park, HoJin Choi, Analysis of Vibration of The Turbine Blades Using Non-Intrusive Stress Measurement System, in: ASME 2006 Power Conference, 2006: pp. 1-7.

[11] C. Lawson, P. Ivey, Compressor Blade Tip Timing Using Capacitance Tip Clearance Probes, Proceedings of ASME Turbo Expo. (2003) 1-8.

[12] P. Russhard, Blade tip timing (BTT) uncertainties, AIP Conference Proceedings. 1740 (2016).

[13] M. Mohamed, P. Bonello, P. Russhard, The Determination Of Steady-State Movements Using Blade Tip Timing Data, in: Proceedings of ASME Turbo Expo, Oslo, Norway, 2018.

[14] P. Russhard, Blade Tip Timing - Frequently asked Questions, 2012.

[15] O. Jousselin, Development of Blade Tip Timing Techniques in Turbo Machinery, The University of Manchester, 2013.

[16] J.M.B. Clifford Hatcher, JR., Method of determining the location of tip timing sensors during operation, US20150199805 A1, 2015.

[17] D. Kominsky, Rotating Stall Detection Using Optical Measurement of Blade Untwist, US 8,854,626 B2, 2011.

[18] G. Battiato, C.M. Firrone, T.M. Berruti, Forced response of rotating bladed disks: Blade TipTiming measurements, Mechanical Systems and Signal Processing. 85 (2017) 912-926.

[19] P. Russhard, Derived once per rev signal generation for Blade Tip Timing systems, Instrumentation Symposium 2014, IET \& ISA 60th .... (2014) 1-5.

[20] A. Savitzky, M.J.E. Golay, Smoothing and Differentiation of Data by Simplified Least Squares Procedures, Analytical Chemistry. 36 (1964) 1627-1639.

[21] I.B. Carrington, Development of Blade Tip Timing Data Analysis Techniques, University of Manchester, 2002. 
[22] J. Gallego-Garrido, G. Dimitriadis, J.R. Wright, Development of a multiple modes simulator of rotating bladed assemblies for blade tip-timing data analysis, Proceedings of the 2002 International Conference on Noise and Vibration Engineering. (2002) 1437-1446.

[23] G. Dimitriadis, I.B. Carrington, J.R. Wright, J.E. Cooper, Blade-Tip Timing Measurement of Synchronous Vibrations of Rotating Bladed Assemblies, Mechanical Systems and Signal Processing. 16 (2002) 599-622.

[24] V. Kharyton, J. Laine, F. Thouverez, O. Kucher, Simulation of Tip-Timing Measurements of a Cracked Bladed Disk Forced Response, Proceedings of ASME Turbo Expo. (2010) 1-10.

[27] J. Strnad, J. Liska, Diagnostic methods of a bladed disc mode shape evaluation used for shrouded blades in steam turbines, Journal of Physics: Conference Series. 659 (2015). 


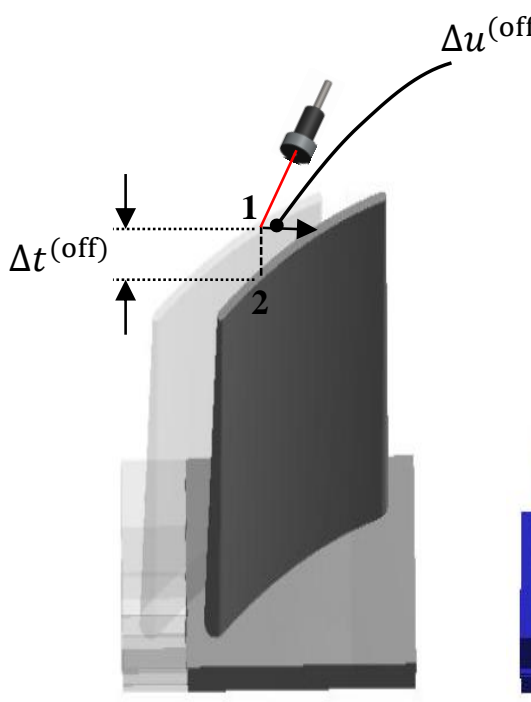

(a)

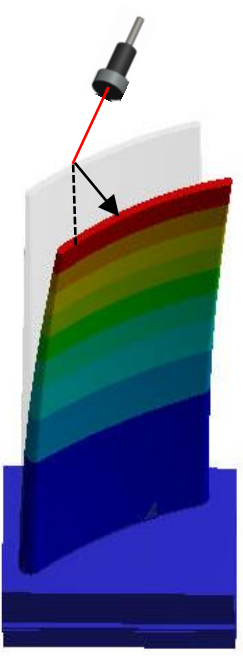

(b)

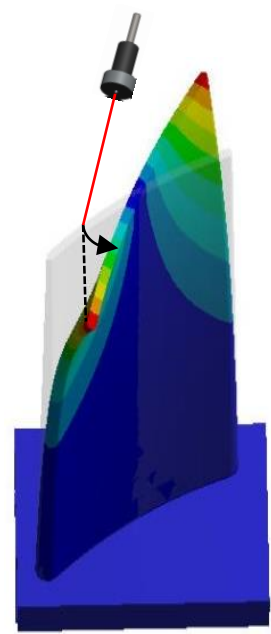

(c)

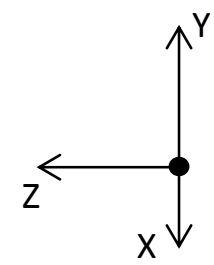

Fig. 1. Steady movements of the blade tip: (a) Axial, (b) Lean, (c) Untwist (the $x-y$ plane is the plane of rotation). 
(a)

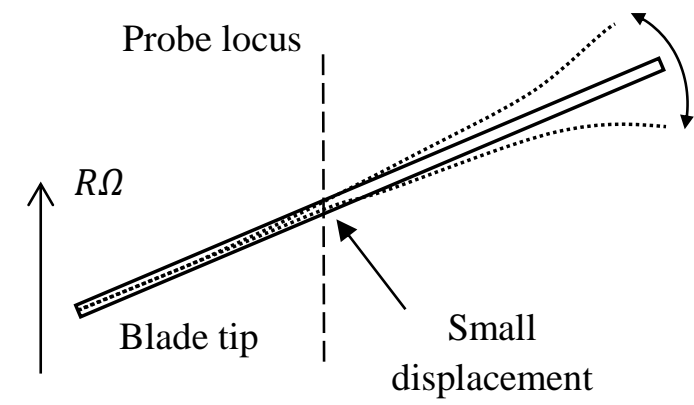

(b)

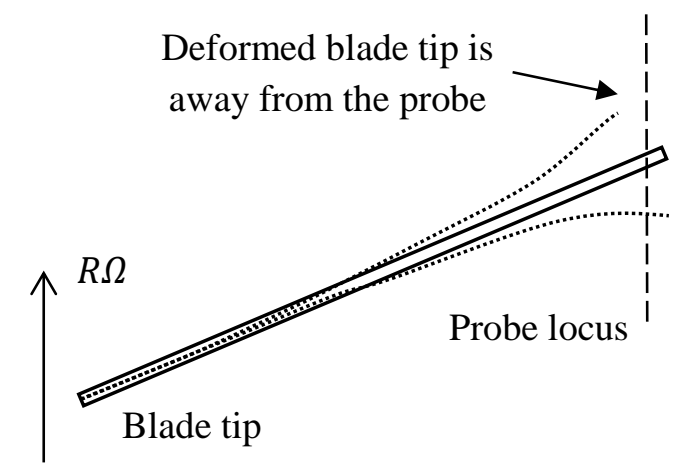

Fig. 2. Misleading positions of the probes: (a) The probe is placed at a position of small displacement; (b) the probe is placed at far edge of blade tip [13] 


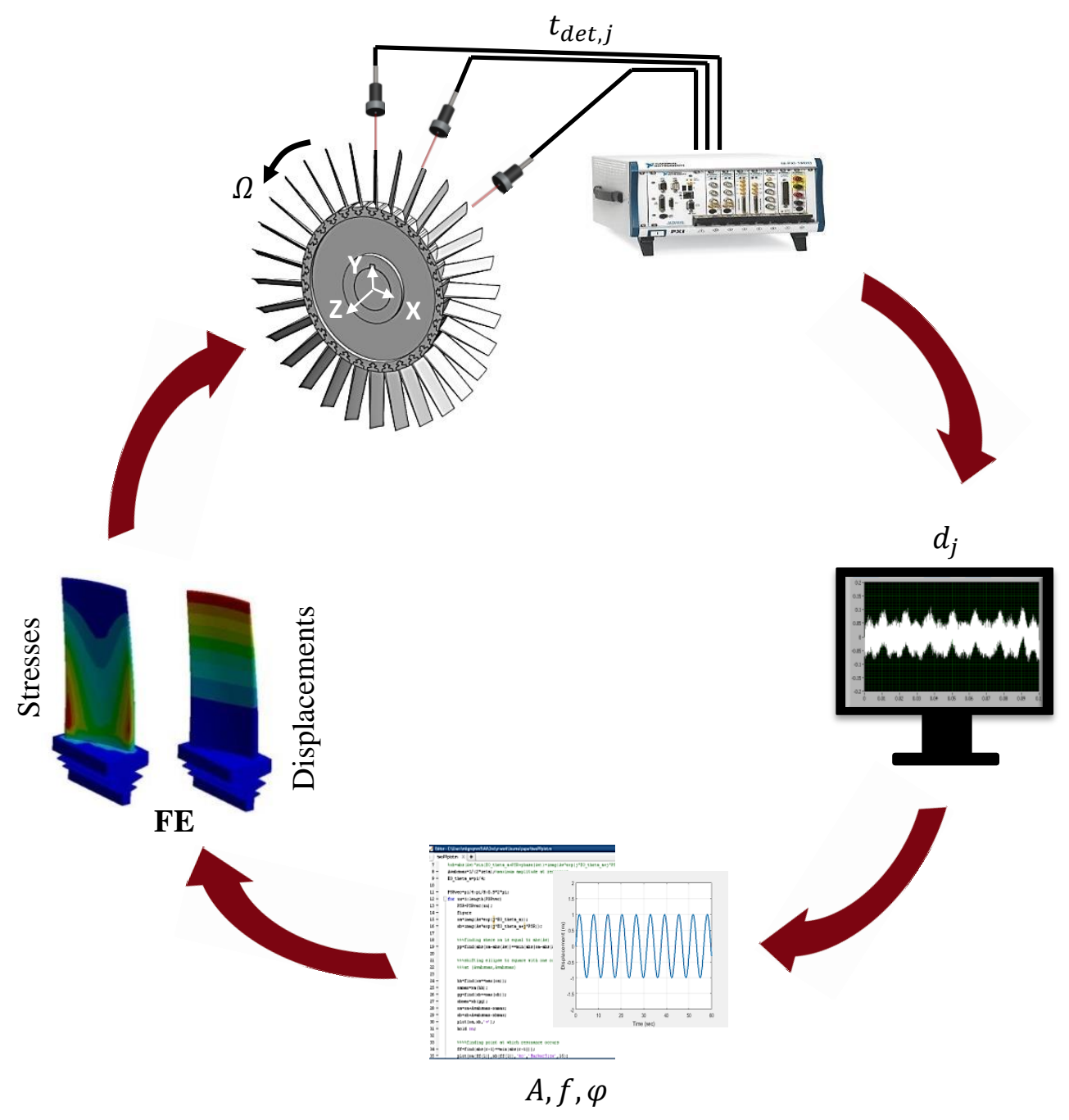

Fig. 3. BTT measurement system $(A, f, \varphi$ respectively denote amplitude, frequency, and phase of vibration) 


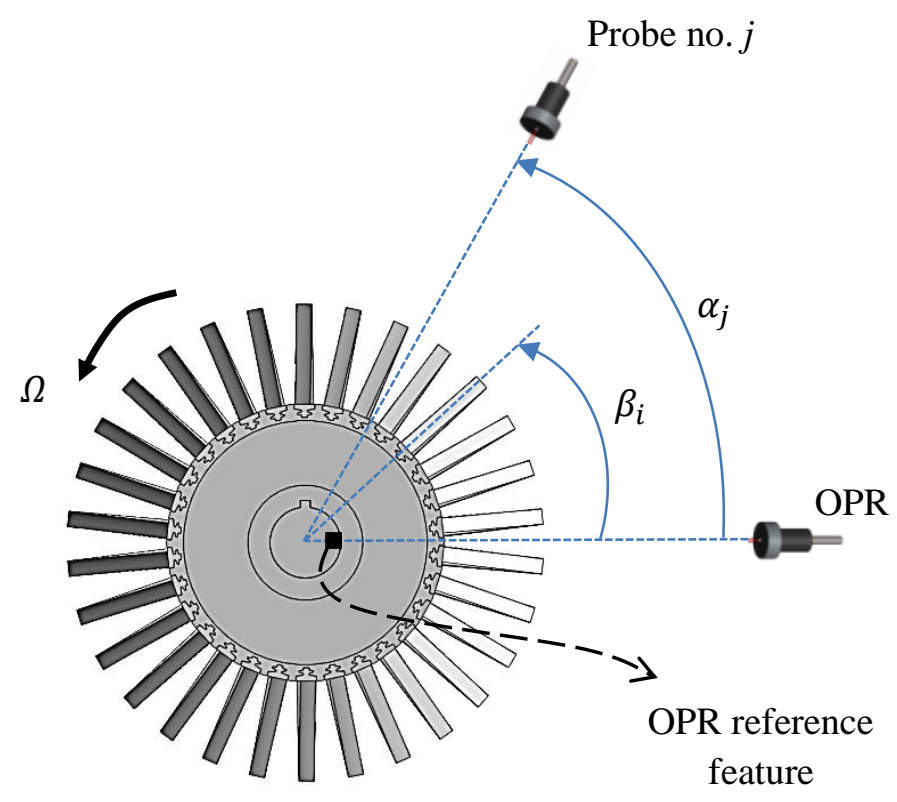

Fig. 4. Typical arrangement for measuring $t_{\mathrm{exp}, j}$ using once-per-revolution (OPR) probe

a)

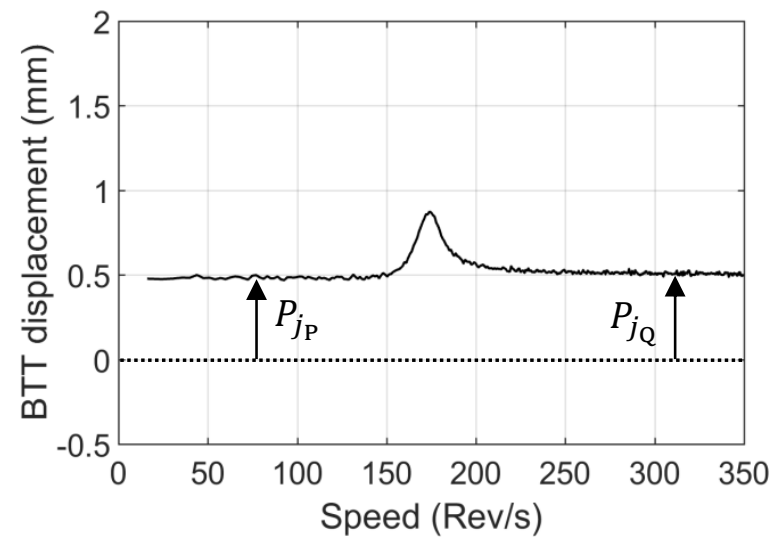

b)

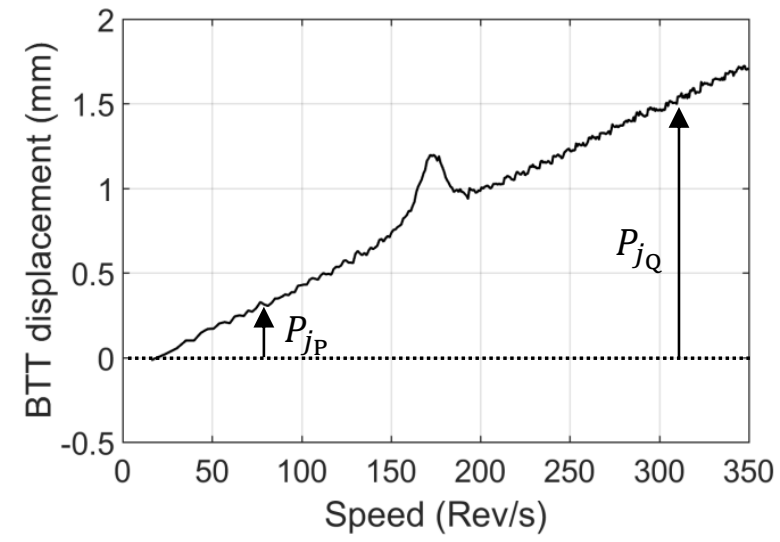

Fig. 5. Effect of steady offset on BTT data: (a) probe or blade positional offset only; (b) speeddependent offset due to speed-dependent steady (non-oscillatory) movement 


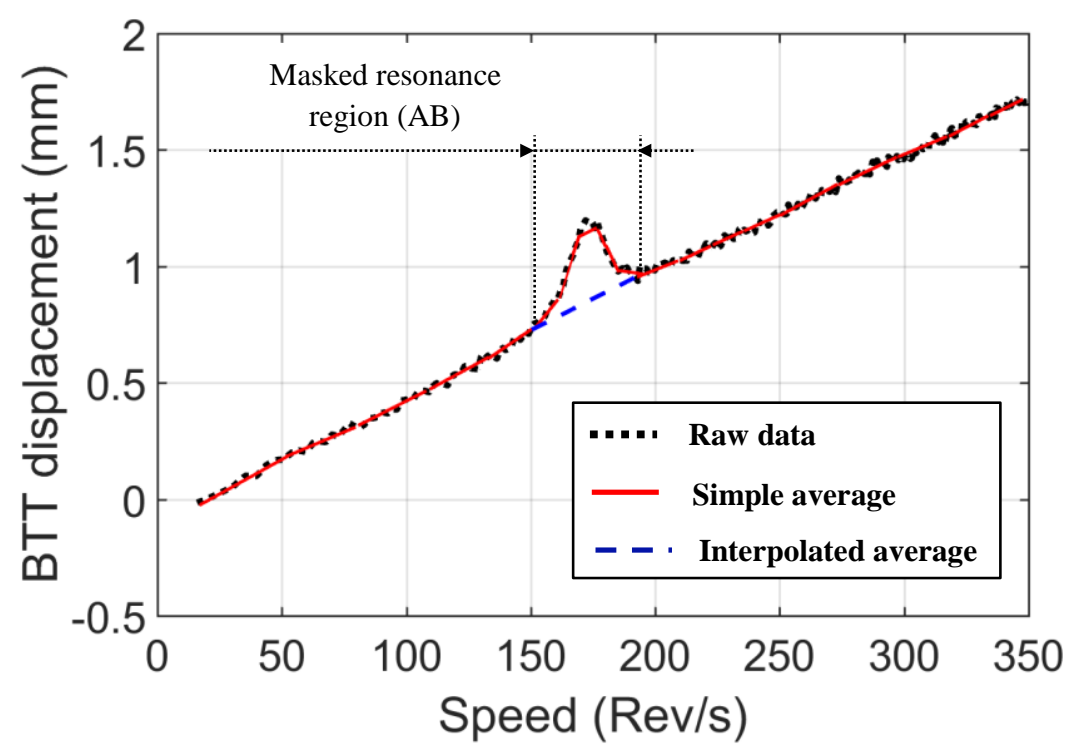

Fig. 6. Extraction of steady offset $P_{j}$ using buffer averaging/interpolated average method of section 4.1

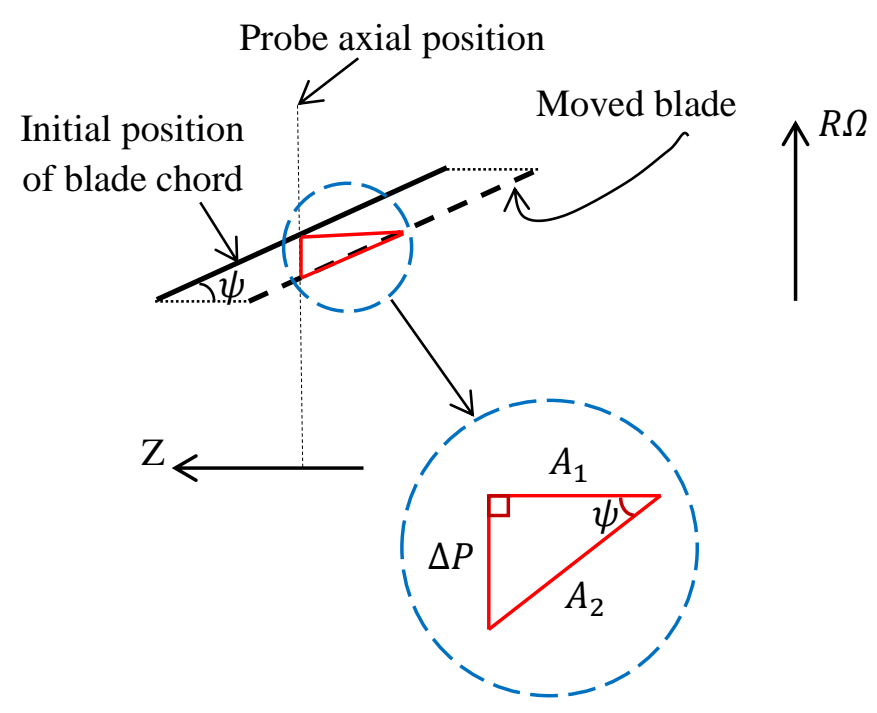

Fig. 7. Geometrical relation for blade axial movement 
Probe axial position

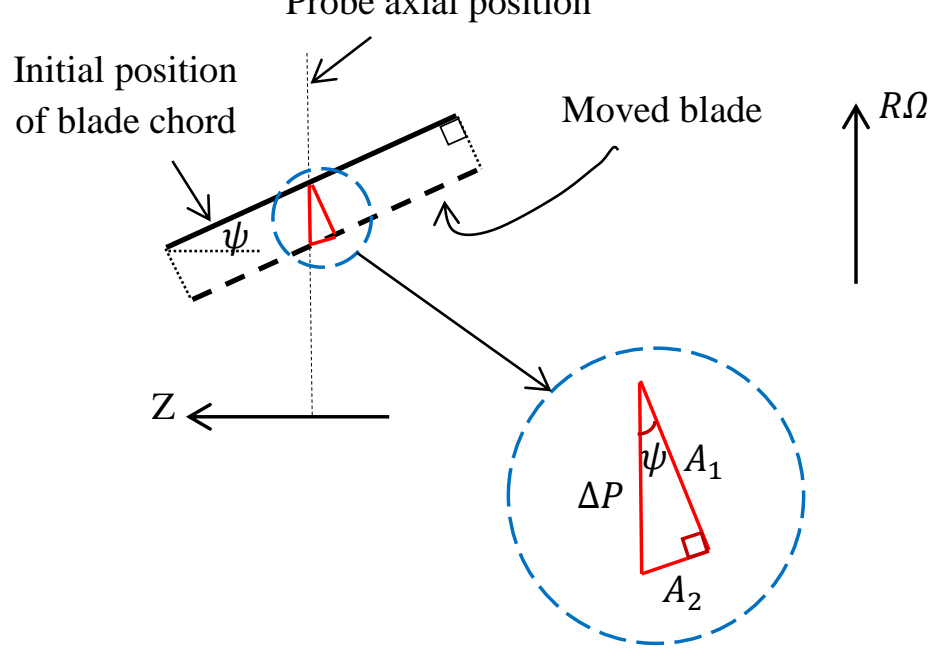

Fig. 8. Geometrical relation for blade lean.

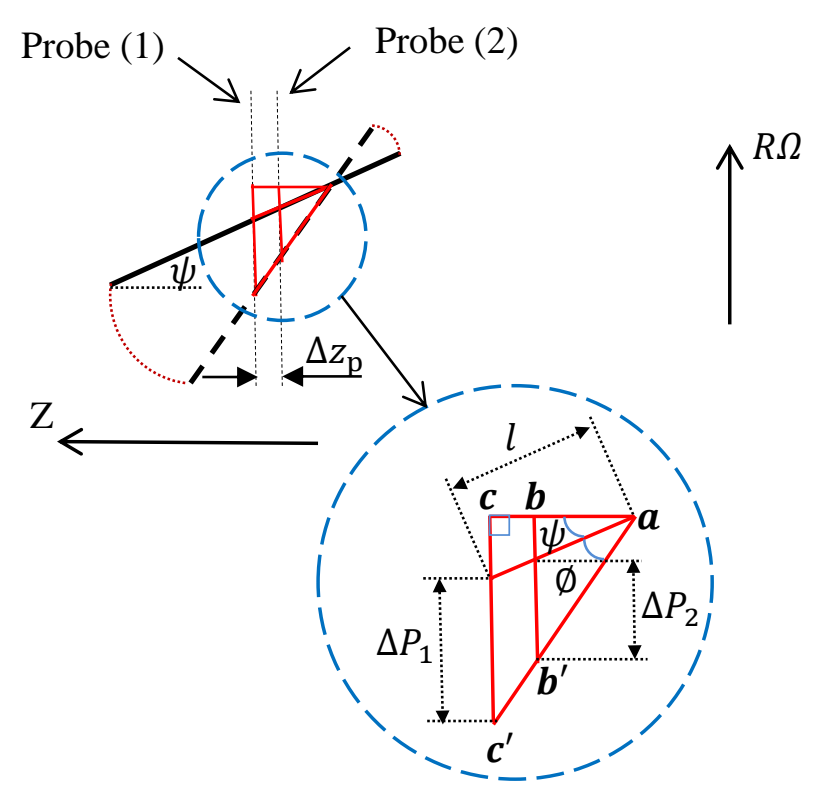

Fig. 9. Geometrical relation for blade untwist. 


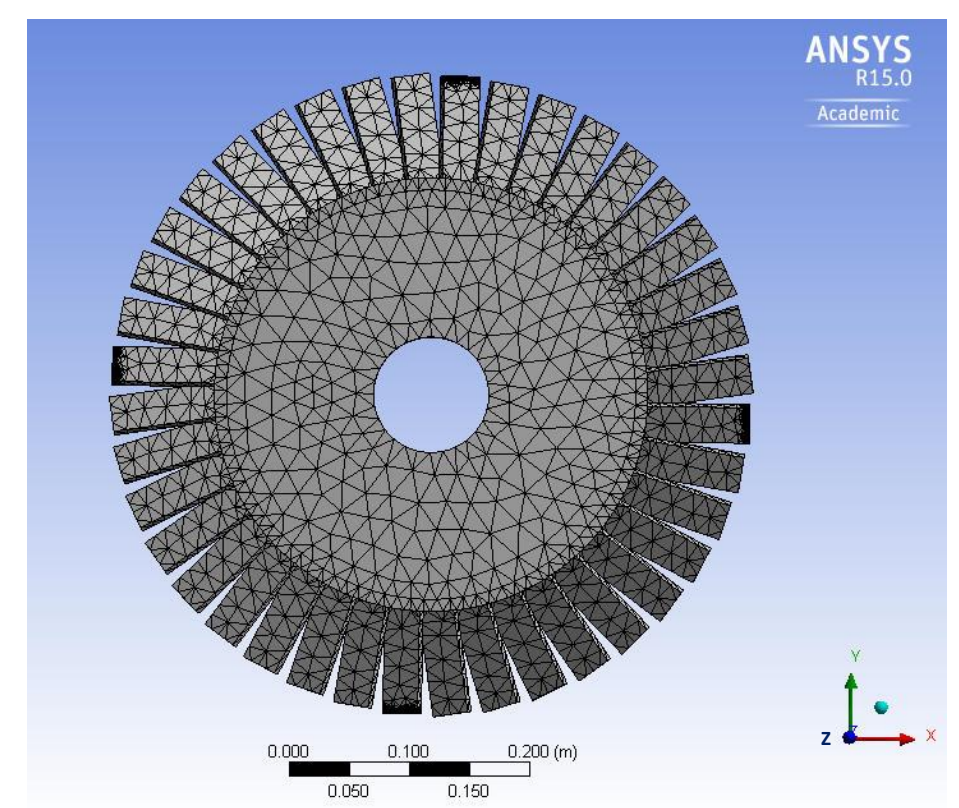

Fig. 10. Blisk FE model.
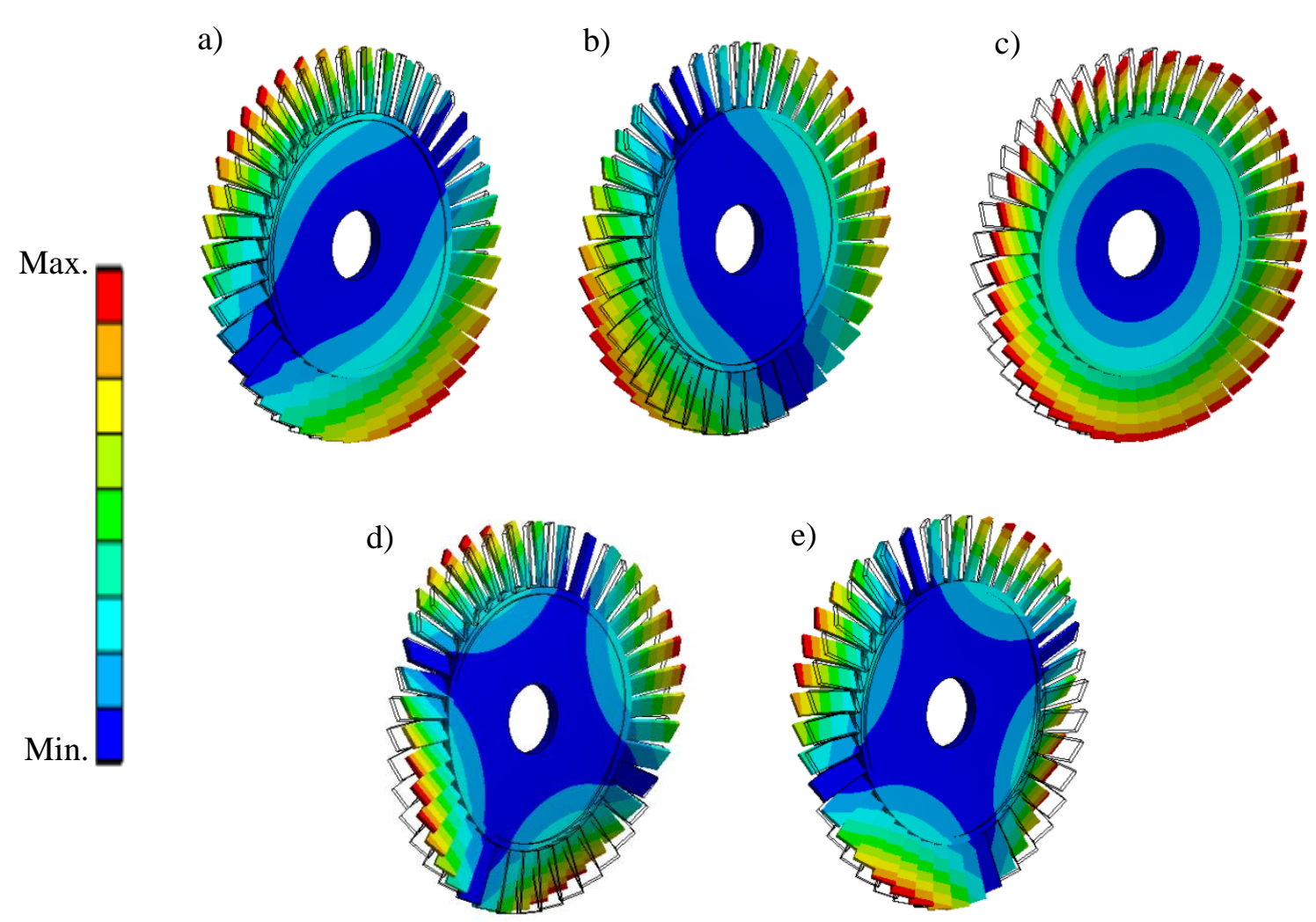

Fig. 11. Blisk mode shapes. a) $1^{\text {st }}$ mode, b) $2^{\text {nd }}$ mode, c) $3^{\text {rd }}$ mode, d) $4^{\text {th }}$ mode, e) $5^{\text {th }}$ mode (corresponding frequencies given in Table 1) 
a)

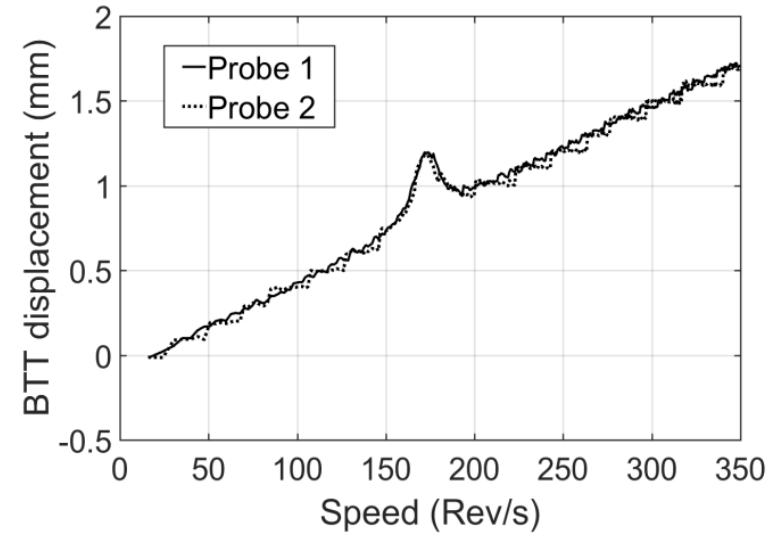

b)

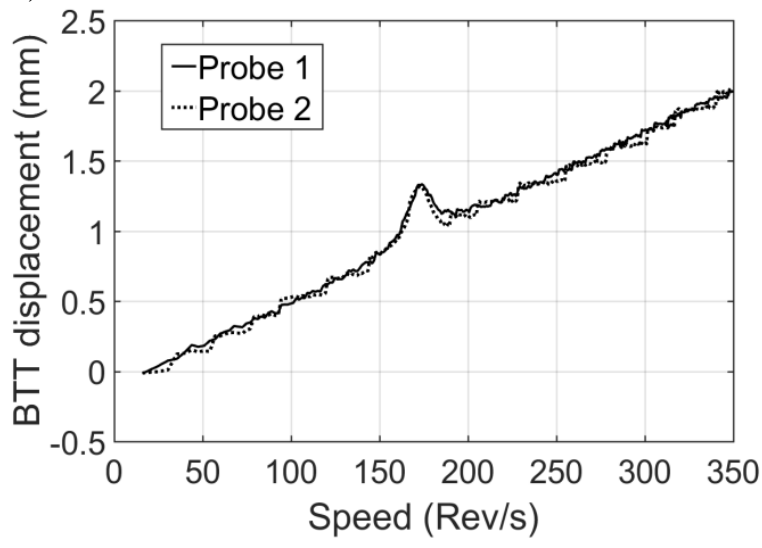

c)

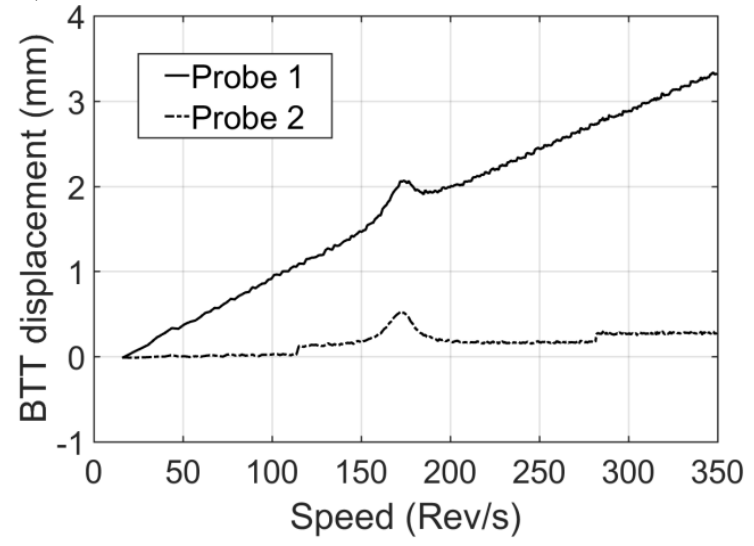

Fig. 12. Simulated BTT data from a given blade, generated by dynamic excitation (travelling wave) with engine order (EO) of 2 and amplitude of $100 \mathrm{~N}$, including three different types of steady movement: (a) axial, (b) lean, (c) untwist. (NB: excitation frequency is twice the speed values along the horizontal axis since $\mathrm{EO}$ is 2) 
a)

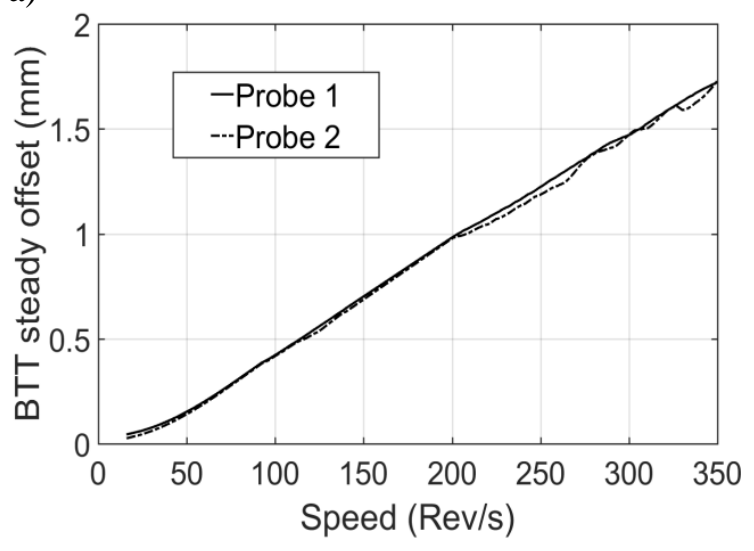

b)

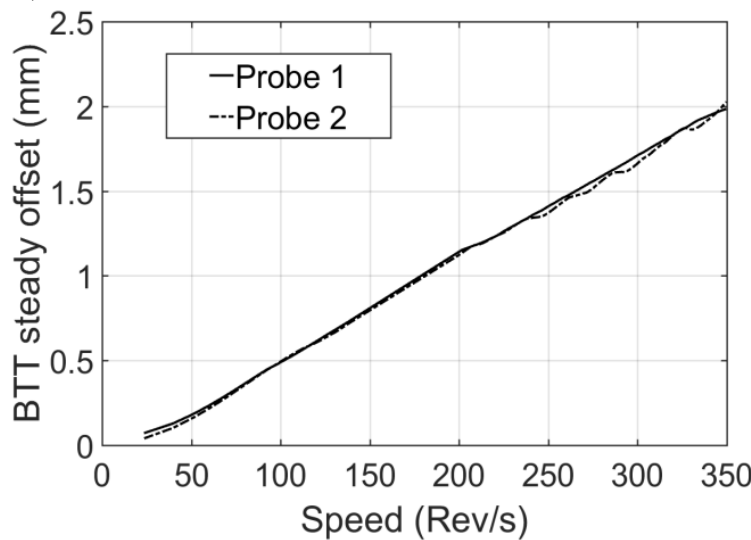

c)

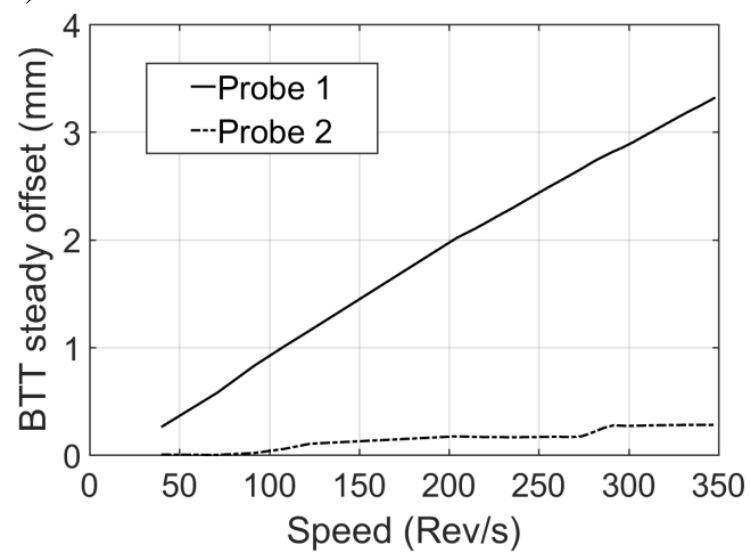

Fig. 13. Variation of steady offset $\left(P_{j}\right)$ computed from the data in Figures $12(\mathrm{a}, \mathrm{b}, \mathrm{c})$ using the buffer average/interpolated average method of section 4.1: (a) axial movement (Figure 12(a)), (b) lean (Figure 12(b)), (c) untwist (Figure 12(c)). (NB: excitation frequency is twice the speed values along the horizontal axis since EO is 2) 


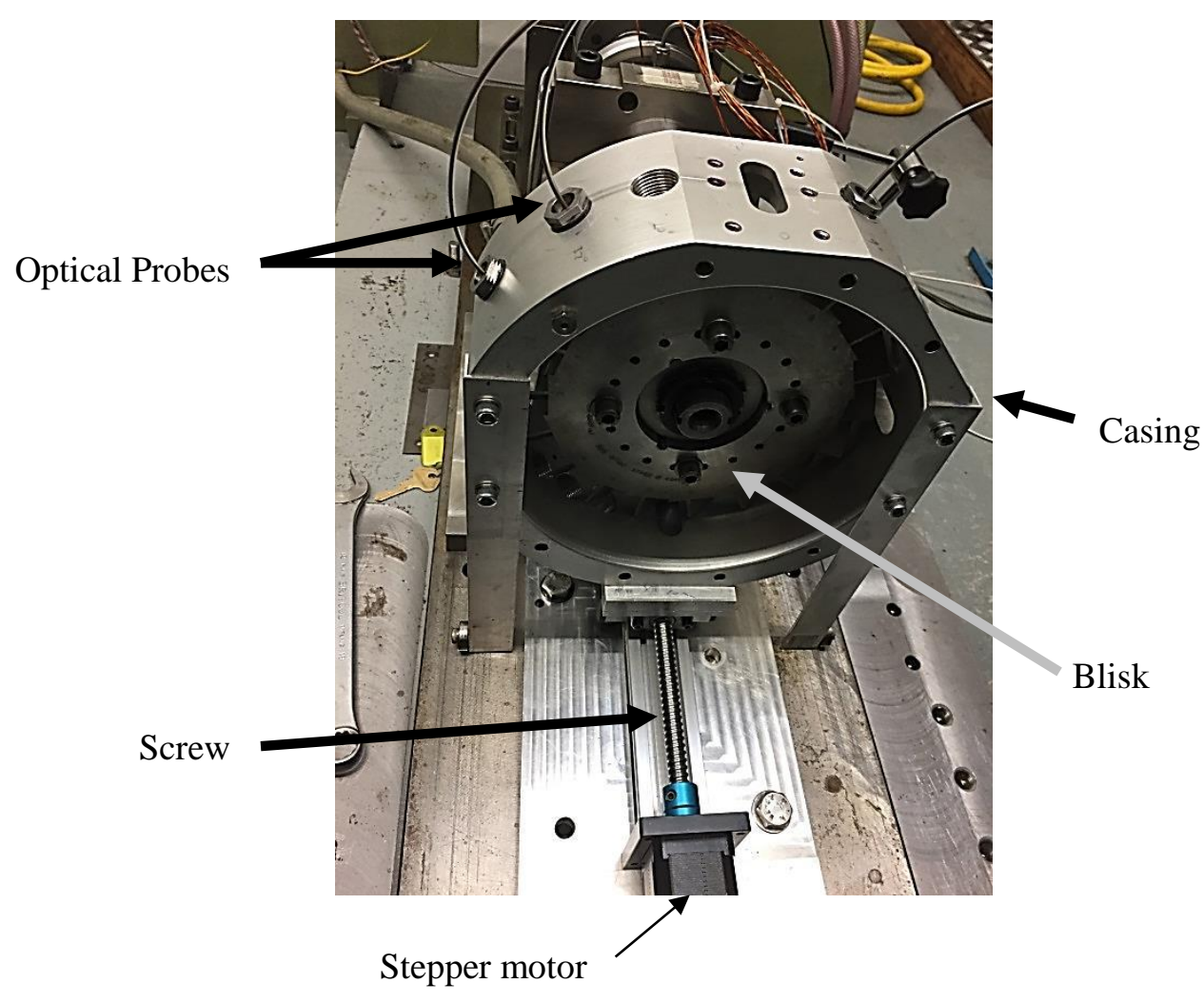

Fig. 14. Test rig with axial movement feature. 
a)

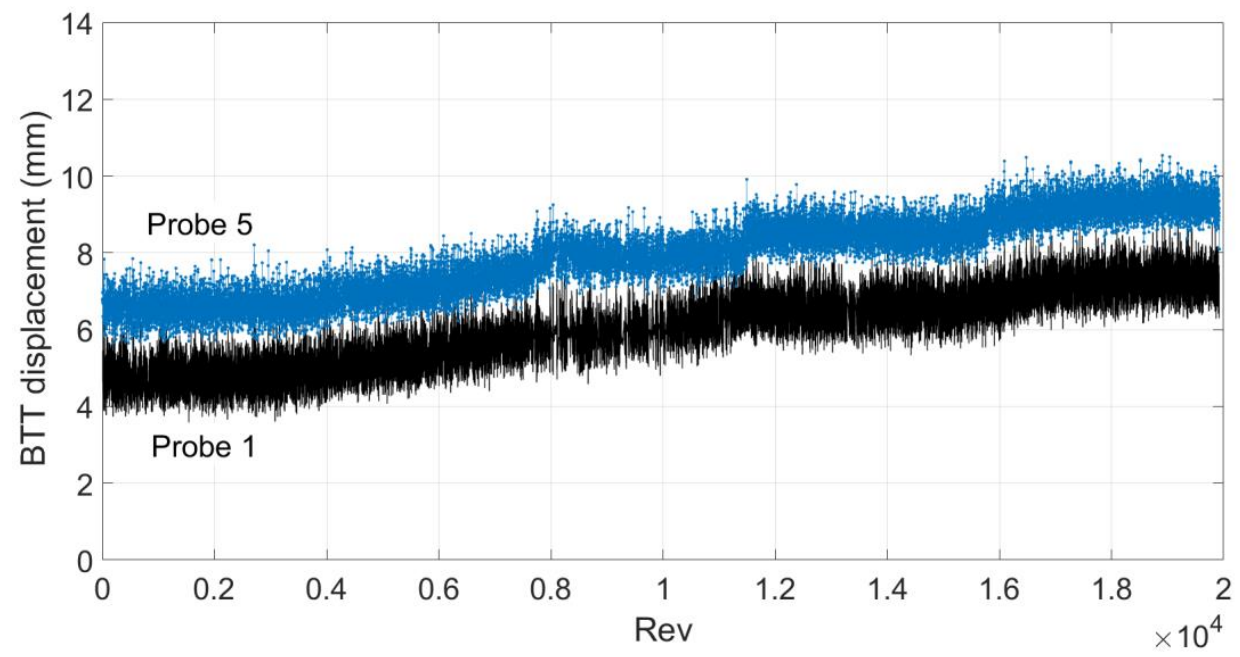

b)

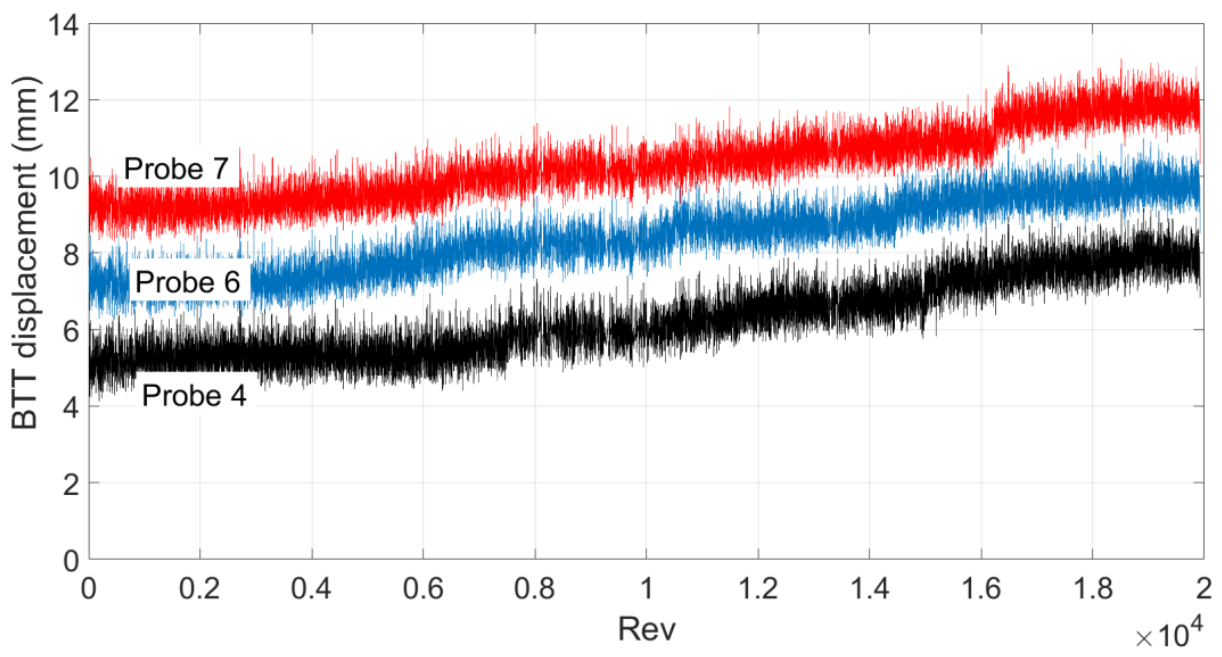

c)

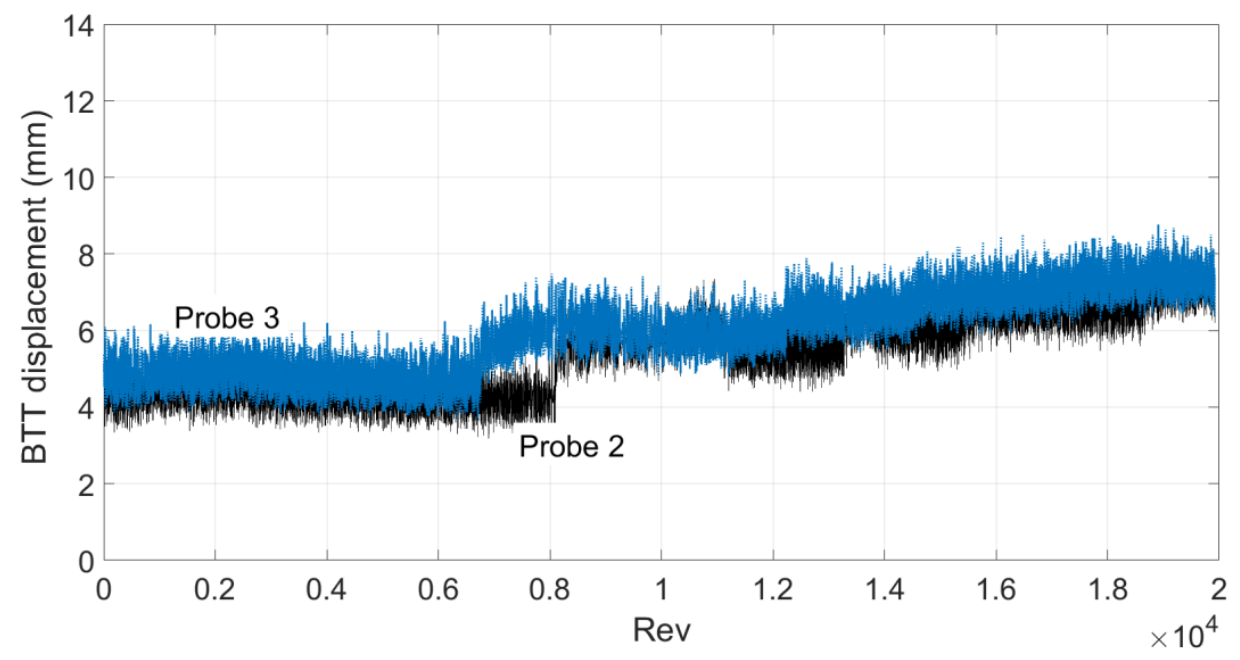

Fig. 15. BTT displacements of a single blade at all probes for first test using test rig (prescribed axial movement of $+3 \mathrm{~mm}$ ): (a) probes 1, 5; (b) probes 4, 6, 7; (c) probes 2, 3 . 


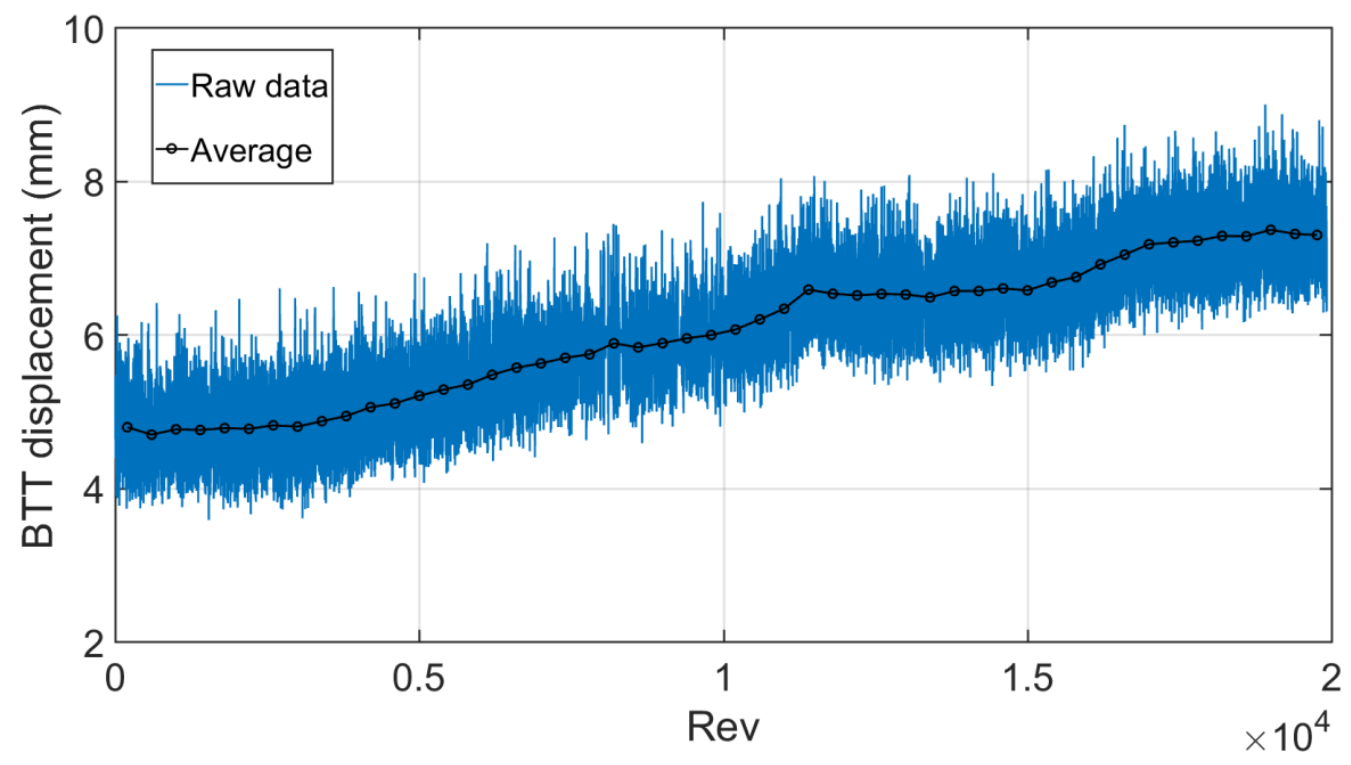

Fig. 16. Averaging the data of a single blade at probe 1 for the steady offset (as per section 4.1) for the first test using test rig (prescribed axial movement of $+3 \mathrm{~mm}$ ).

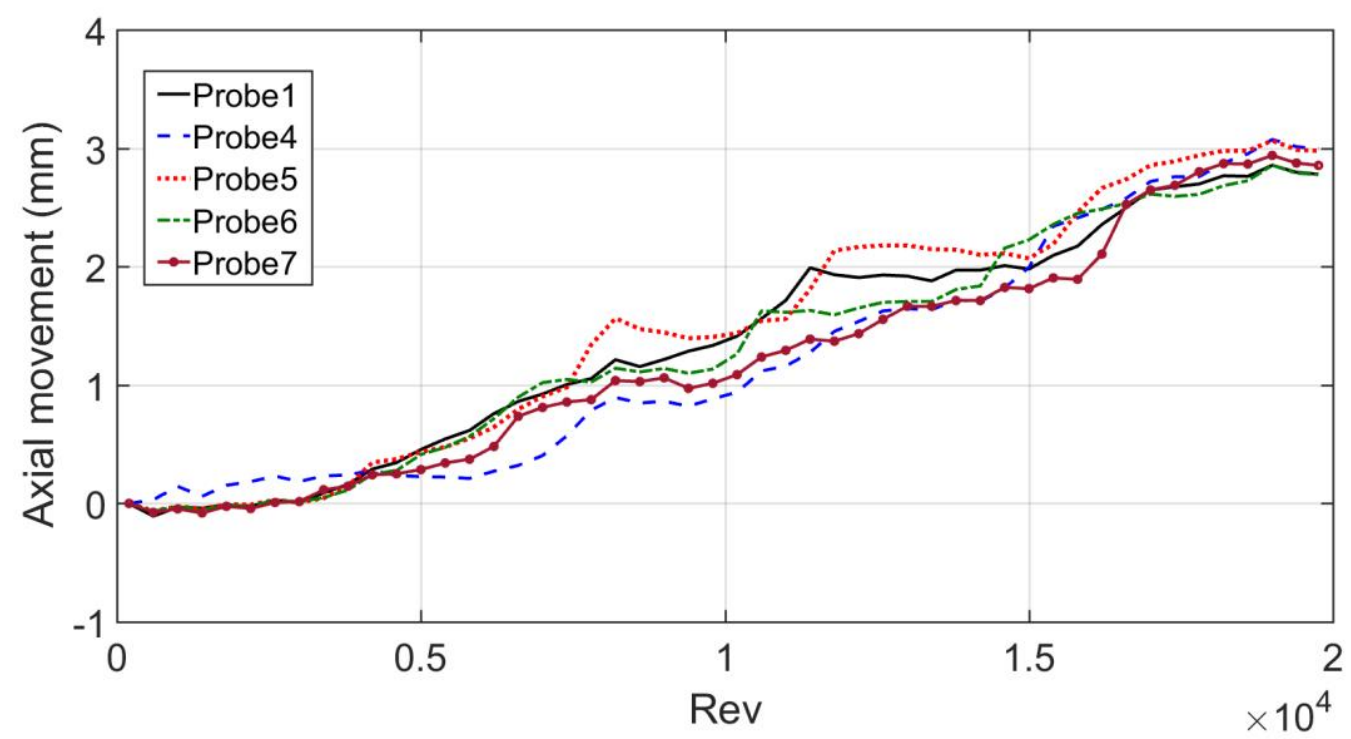

Fig. 17. Calculated axial movement for first test using test rig (prescribed axial movement of $+3 \mathrm{~mm}$ ). 
a)

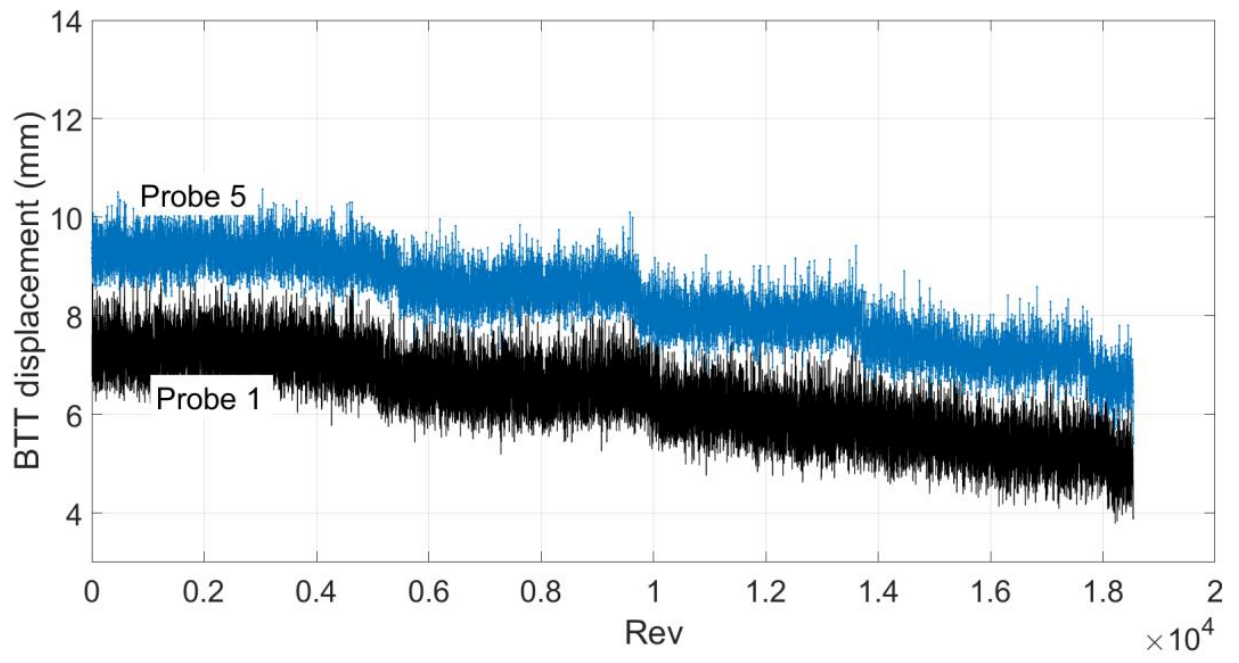

b)

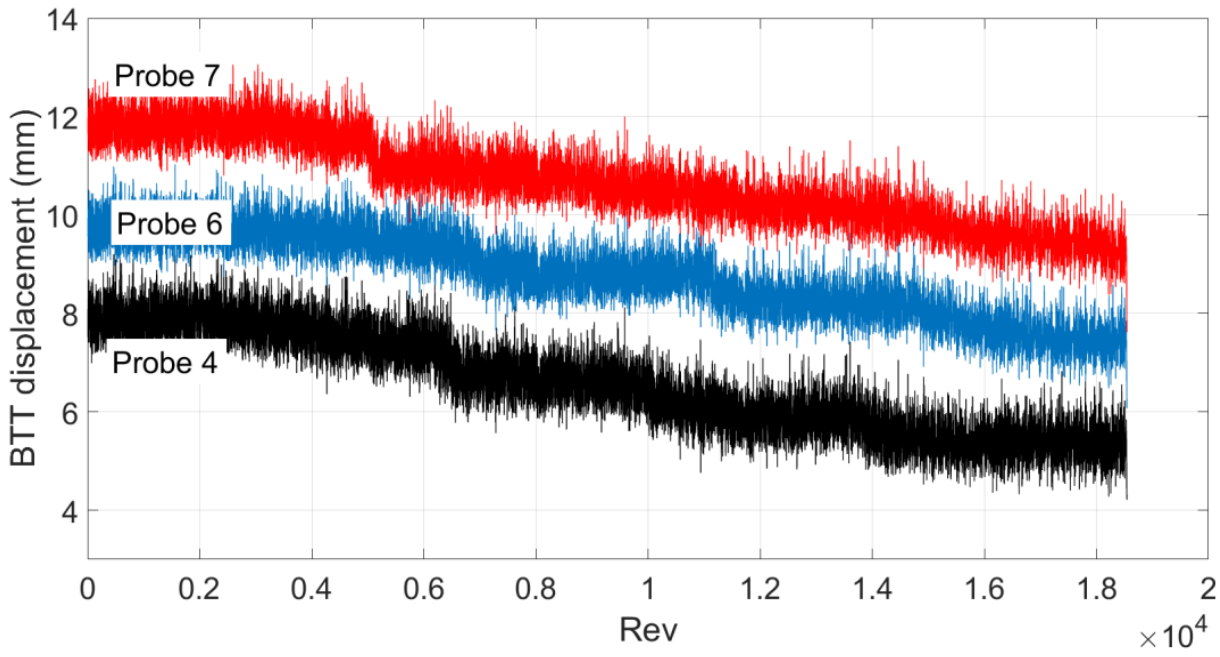

c)

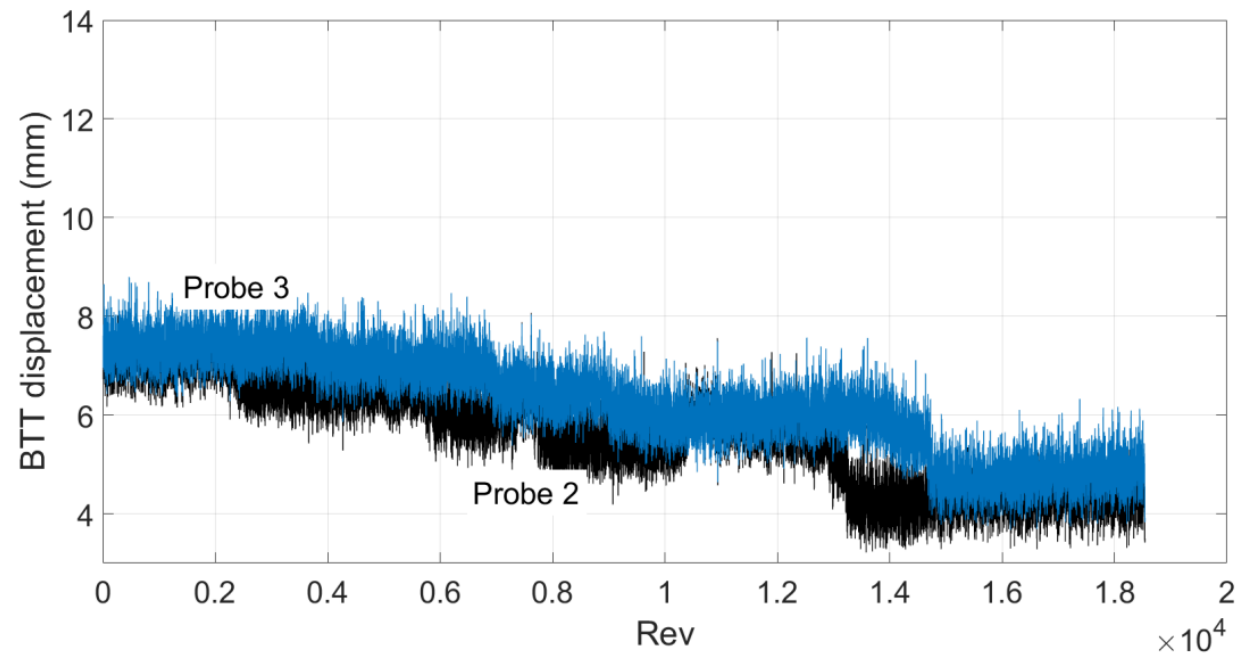

Fig. 18. BTT displacements of a single blade at all probes for second test using test rig (prescribed axial movement of $-3 \mathrm{~mm}$ ): (a) probes 1, 5; (b) probes 4, 6, 7; (c) probes 2, 3 . 


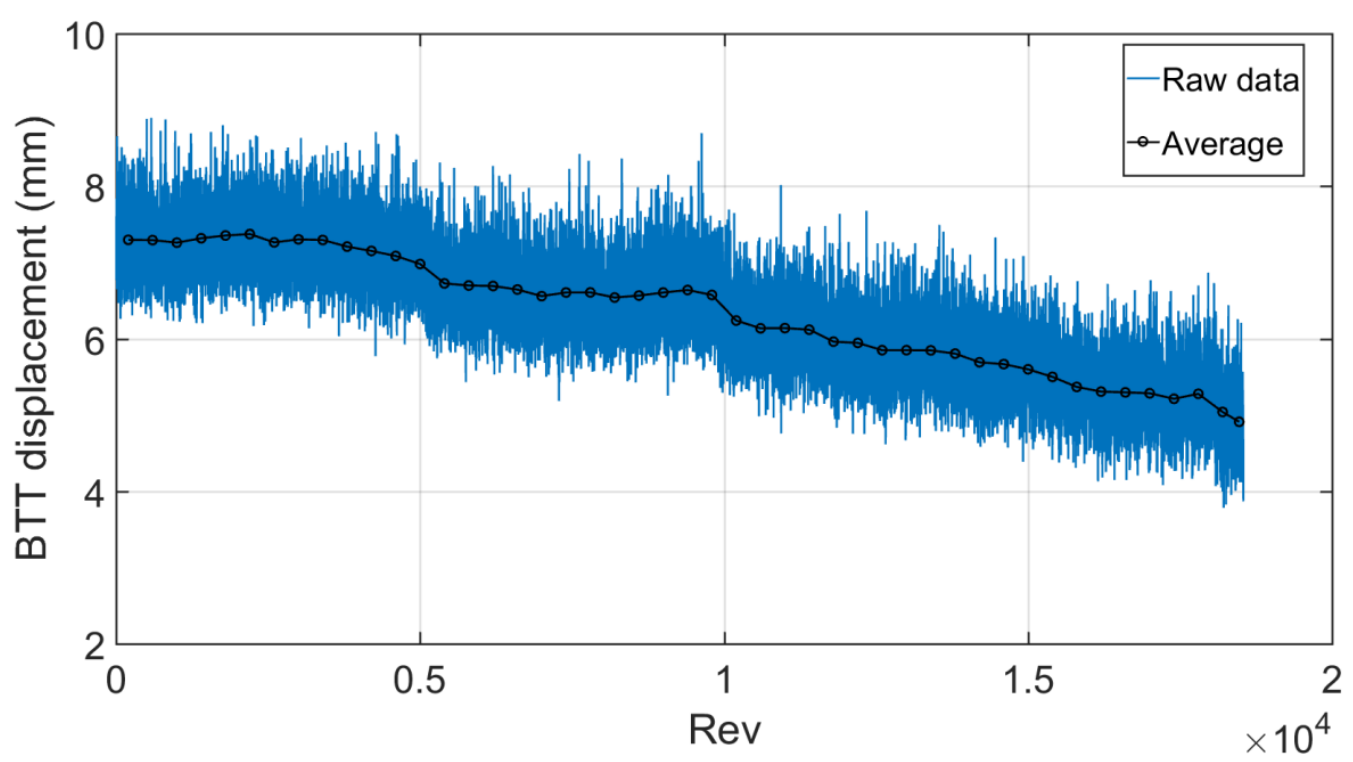

Fig. 19. Averaging the data of a single blade at probe 1 for the steady offset (as per section 4.1) for the second test using test rig (prescribed axial movement of $-3 \mathrm{~mm}$ ).

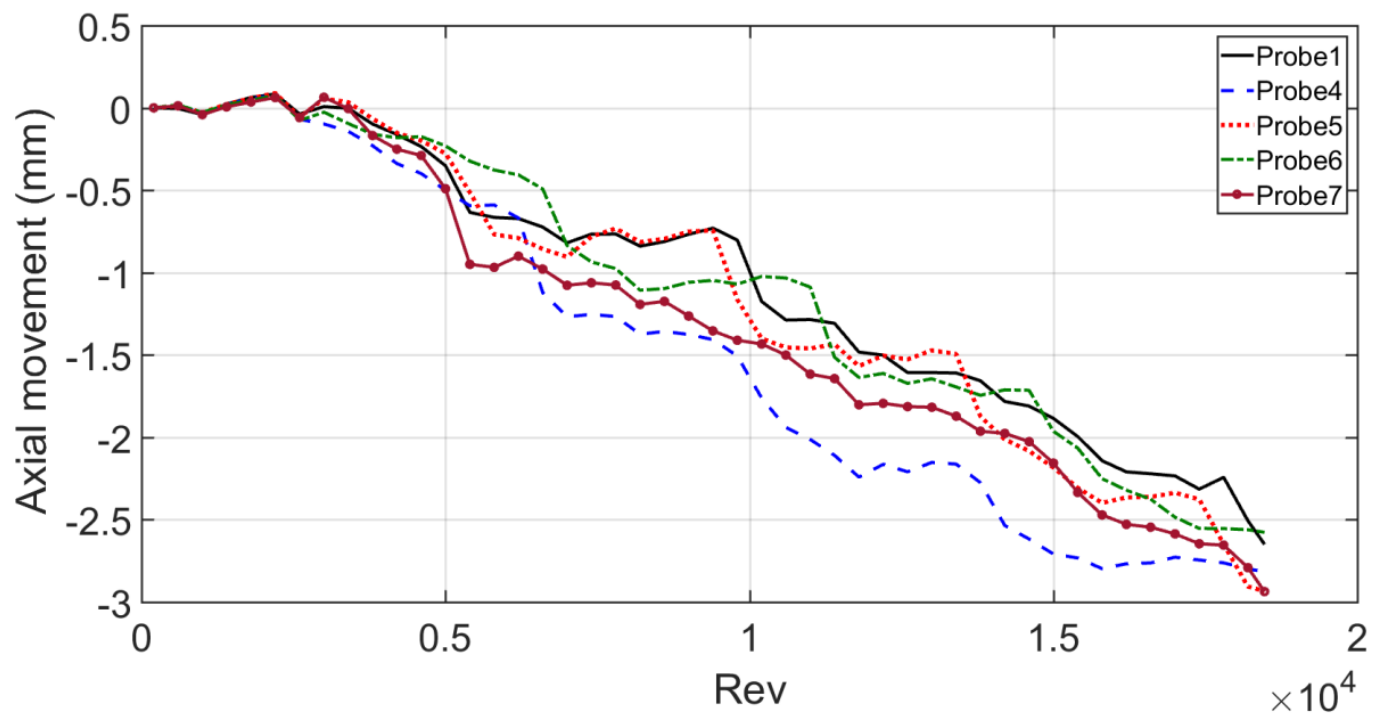

Fig. 20. Calculated axial movement for second test using test rig (prescribed axial movement of $-3 \mathrm{~mm}$ ). 


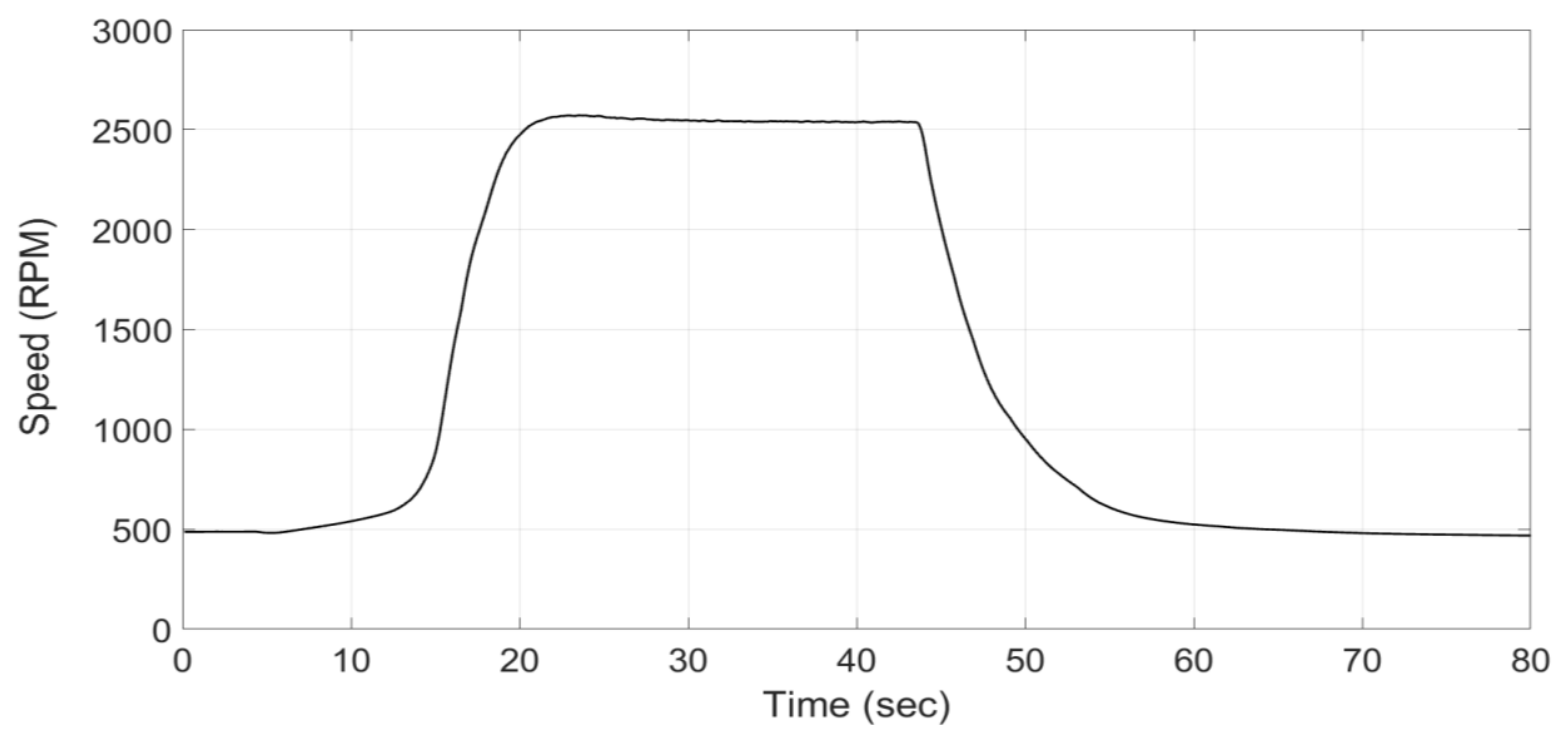

Fig. 21. Variation of speed during the first engine test.

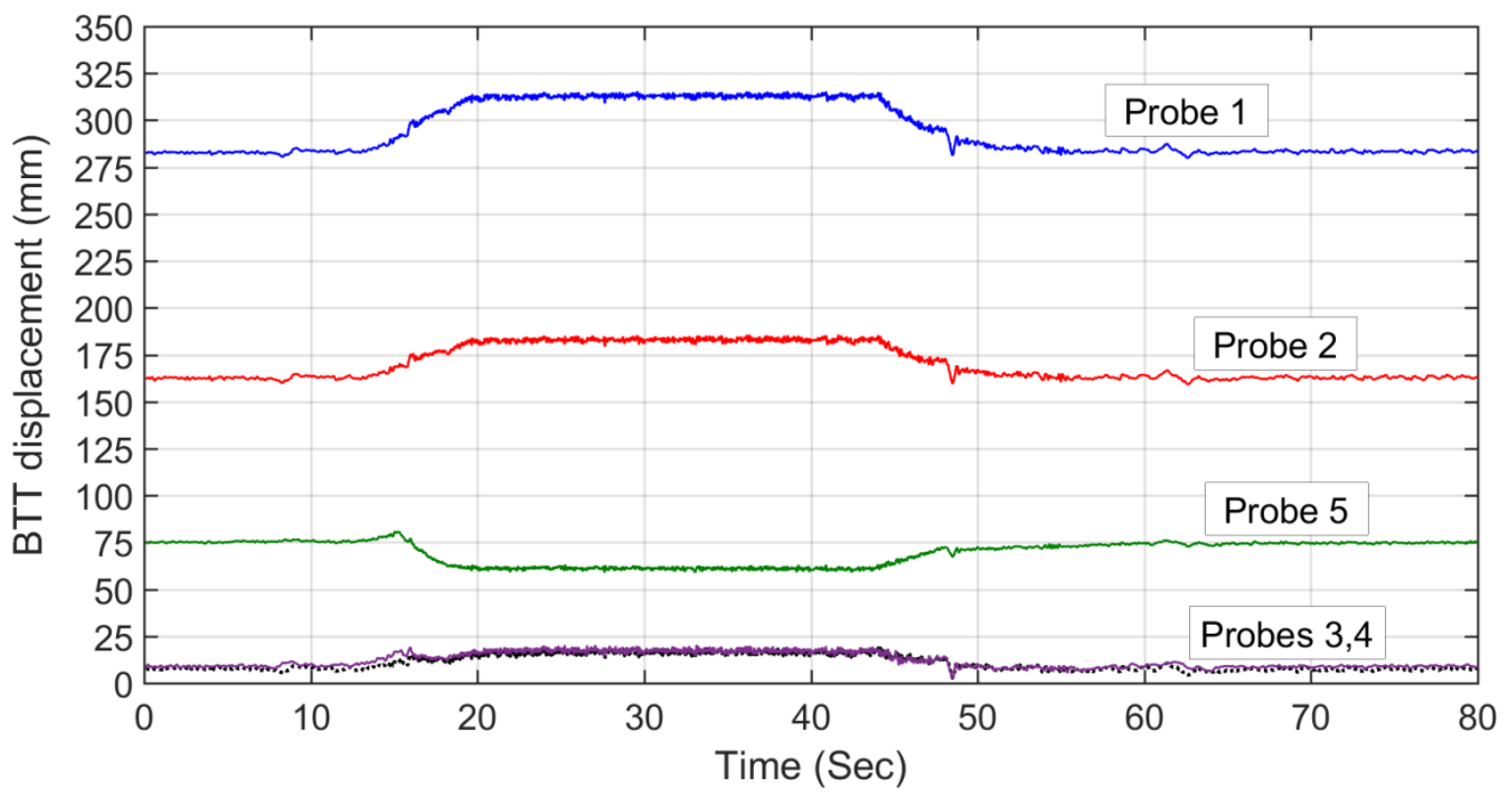

Fig. 22. BTT displacements of a single blade at all probes for the first engine test. 


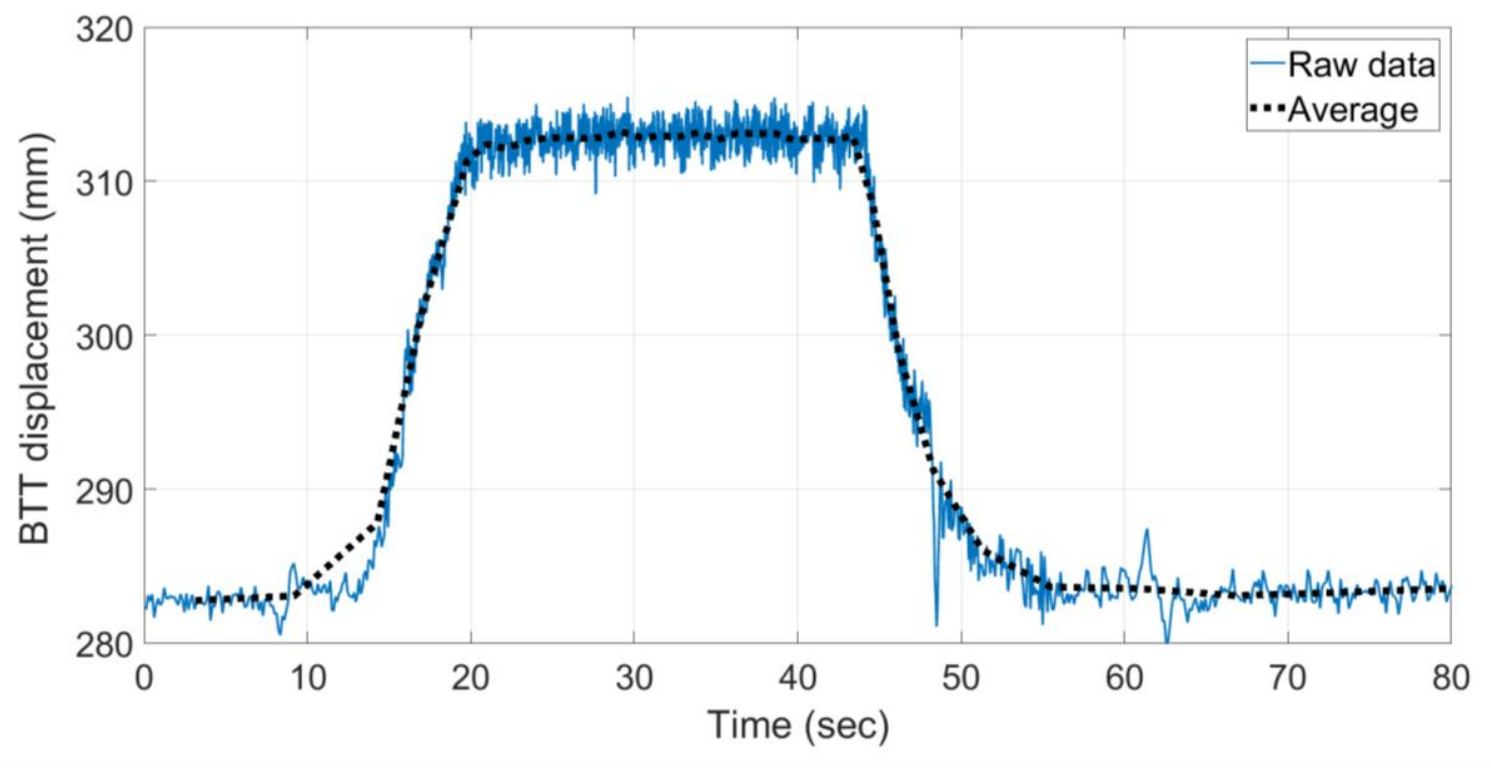

Fig. 23. Averaging the data of a single blade at probe 1 for the steady offset (as per section 4.1) for the first engine test.

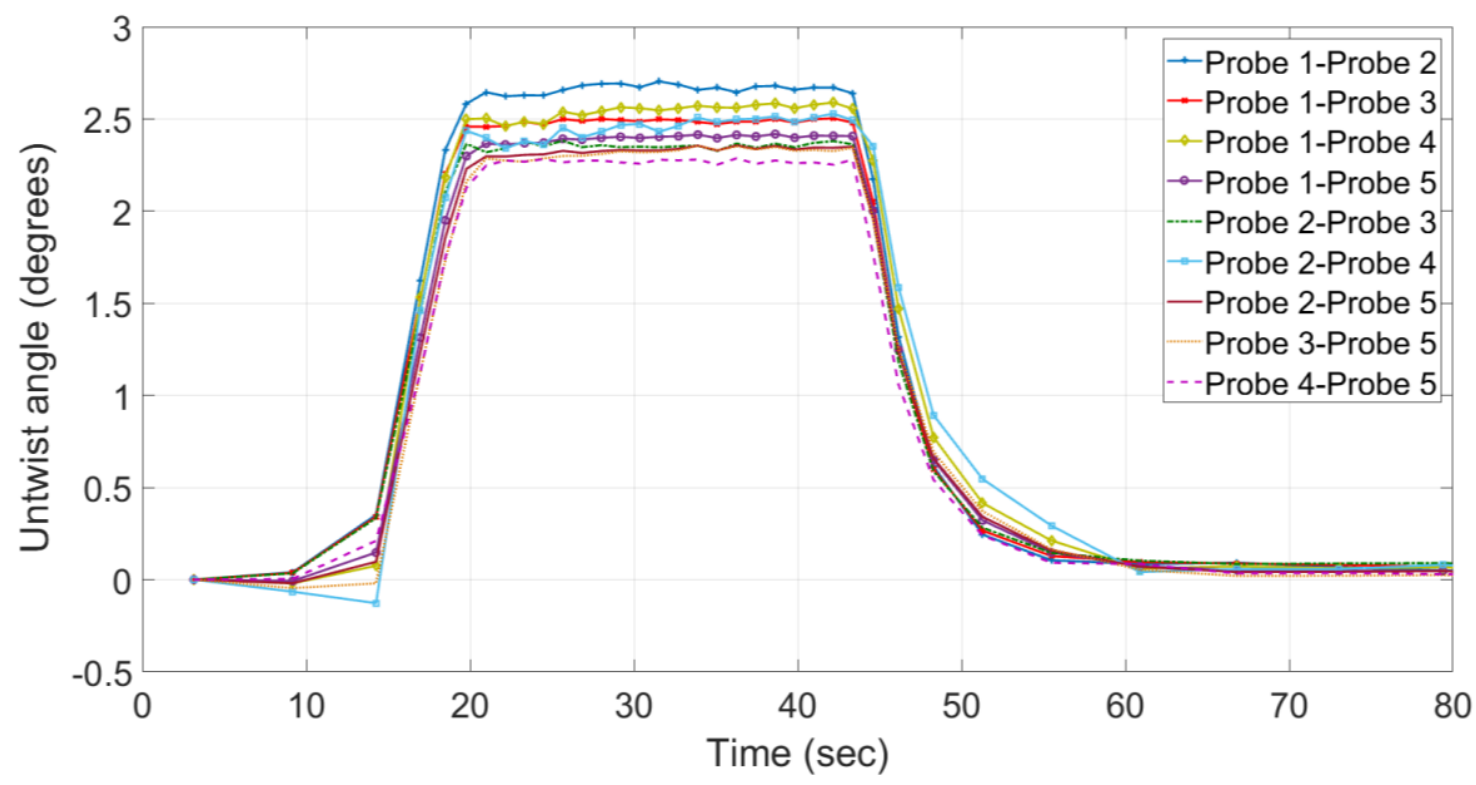

Fig. 24. Variation of untwist angle of a single blade calculated using all combinations of probepairs for the first engine test. 


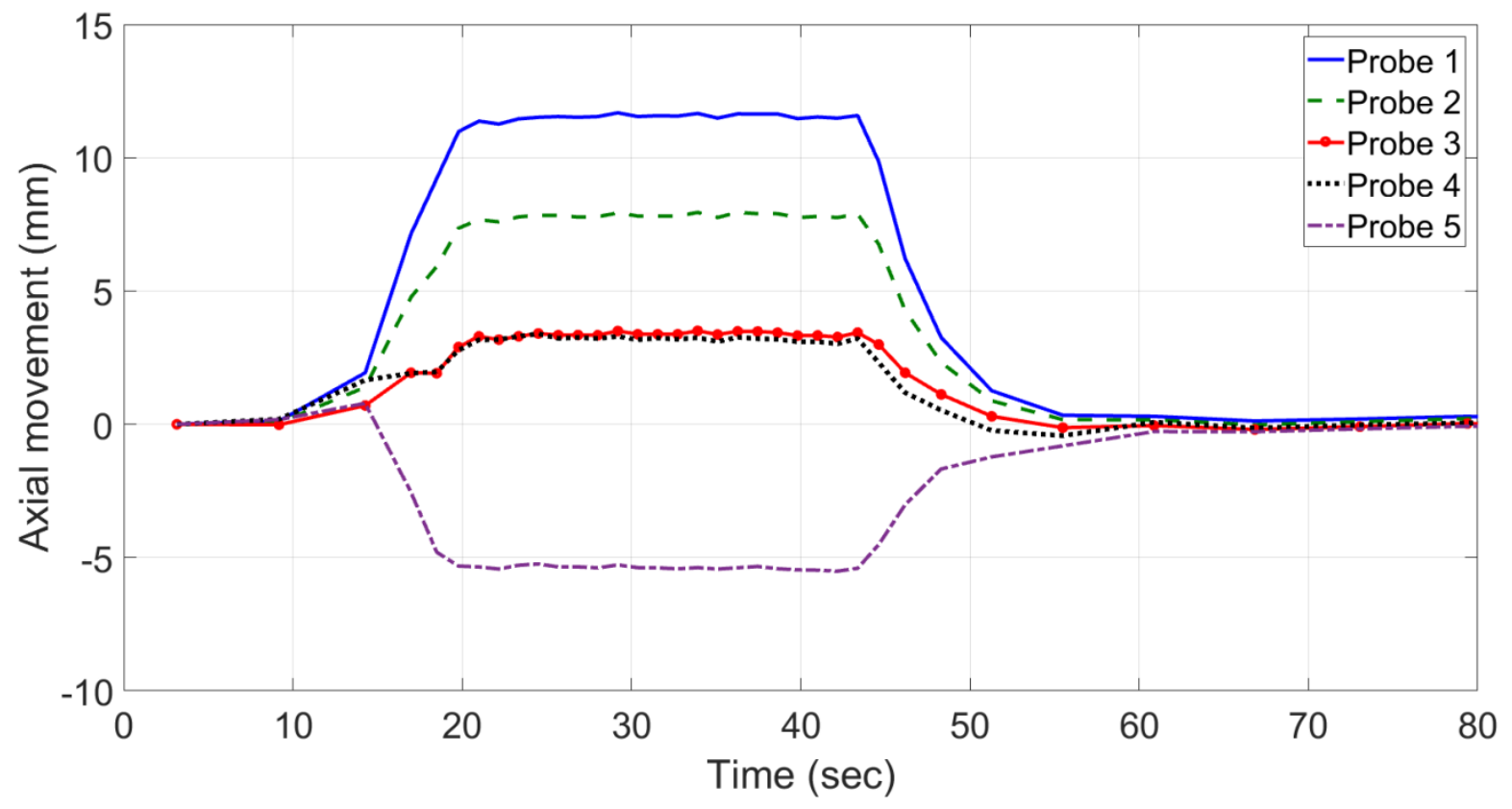

Fig. 25. Variation of the calculated equivalent axial movement for the first engine test.

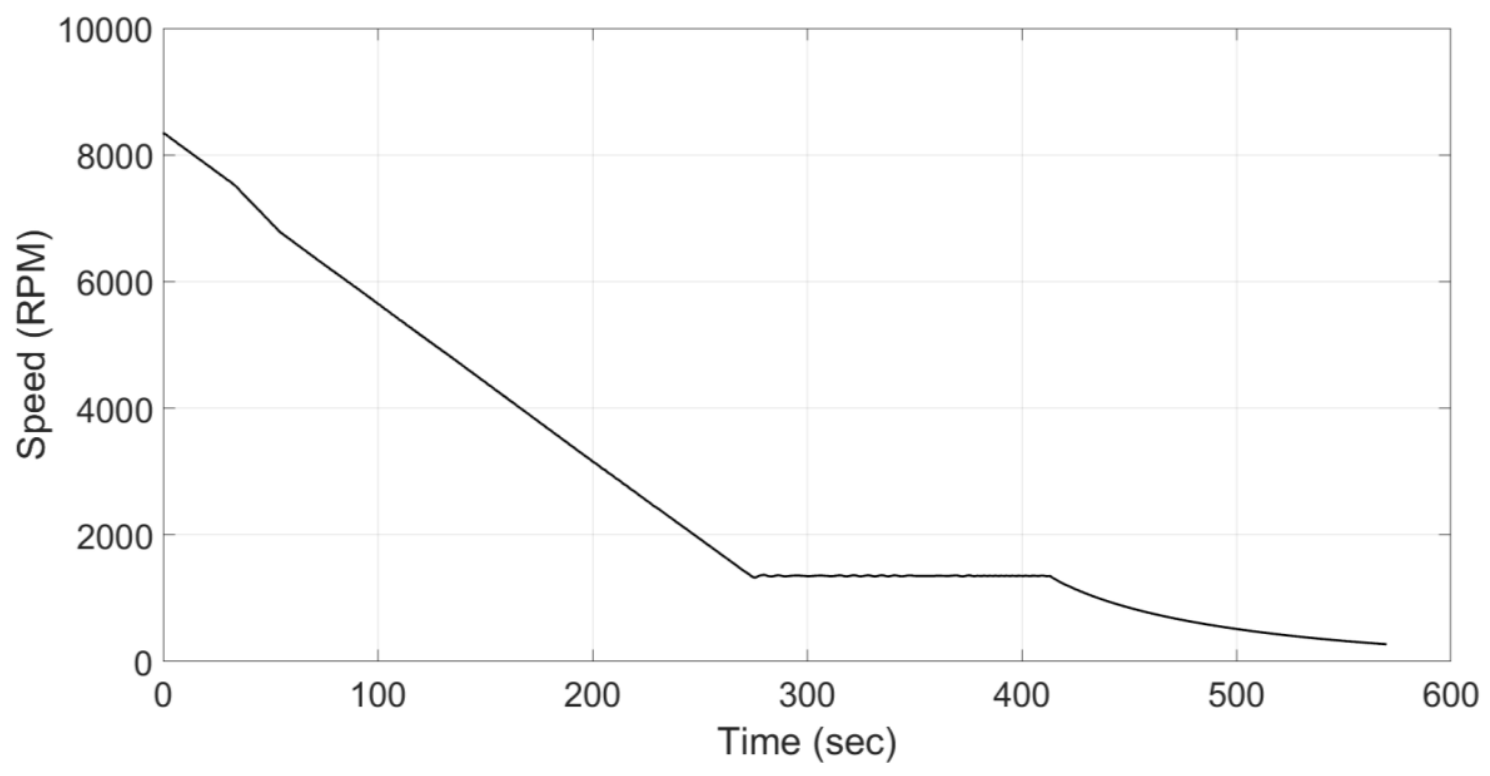

Fig. 26. Variation of speed during the second engine test. 


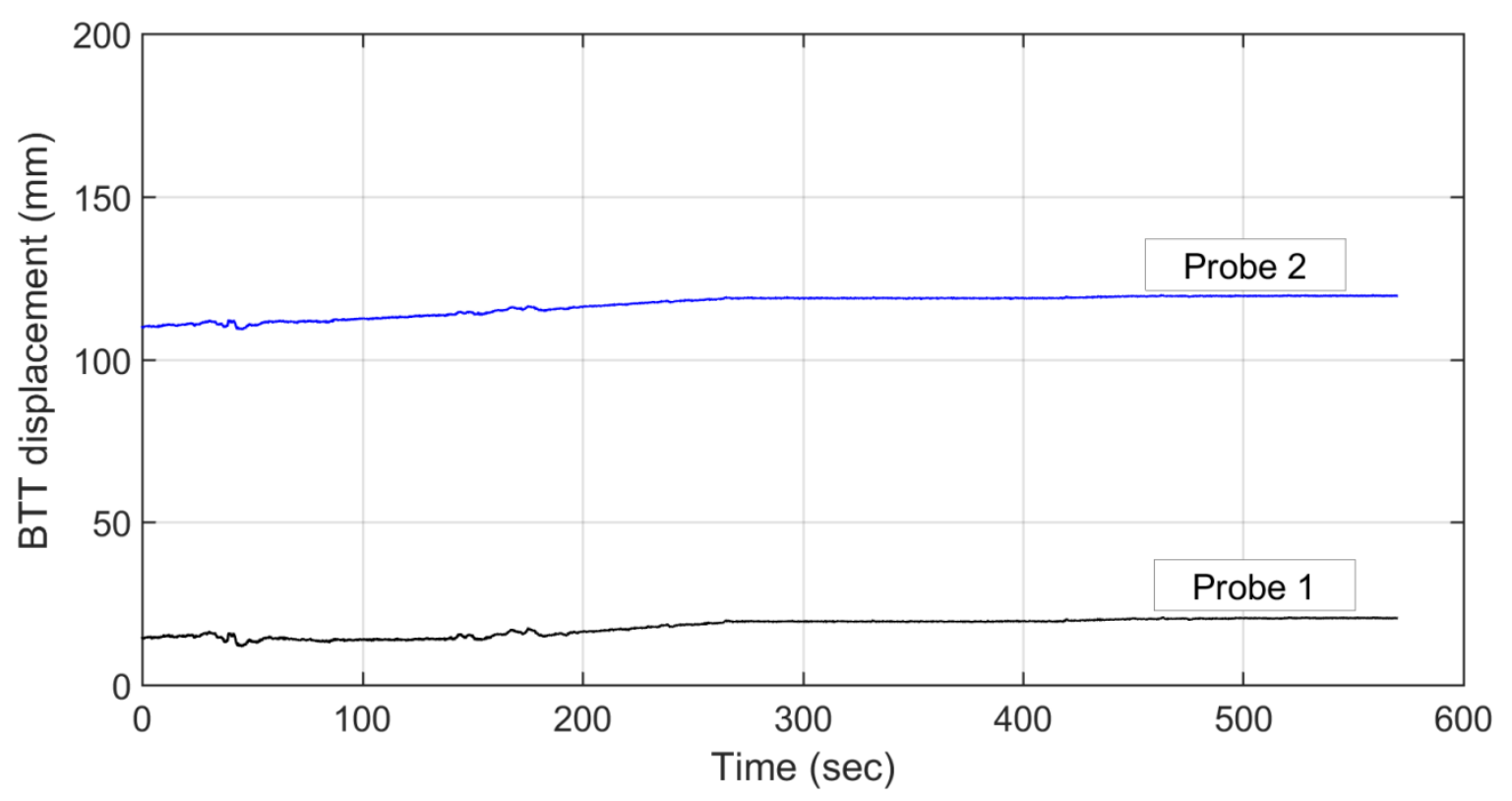

Fig. 27. BTT displacements of a single blade at all probes for the second engine test.

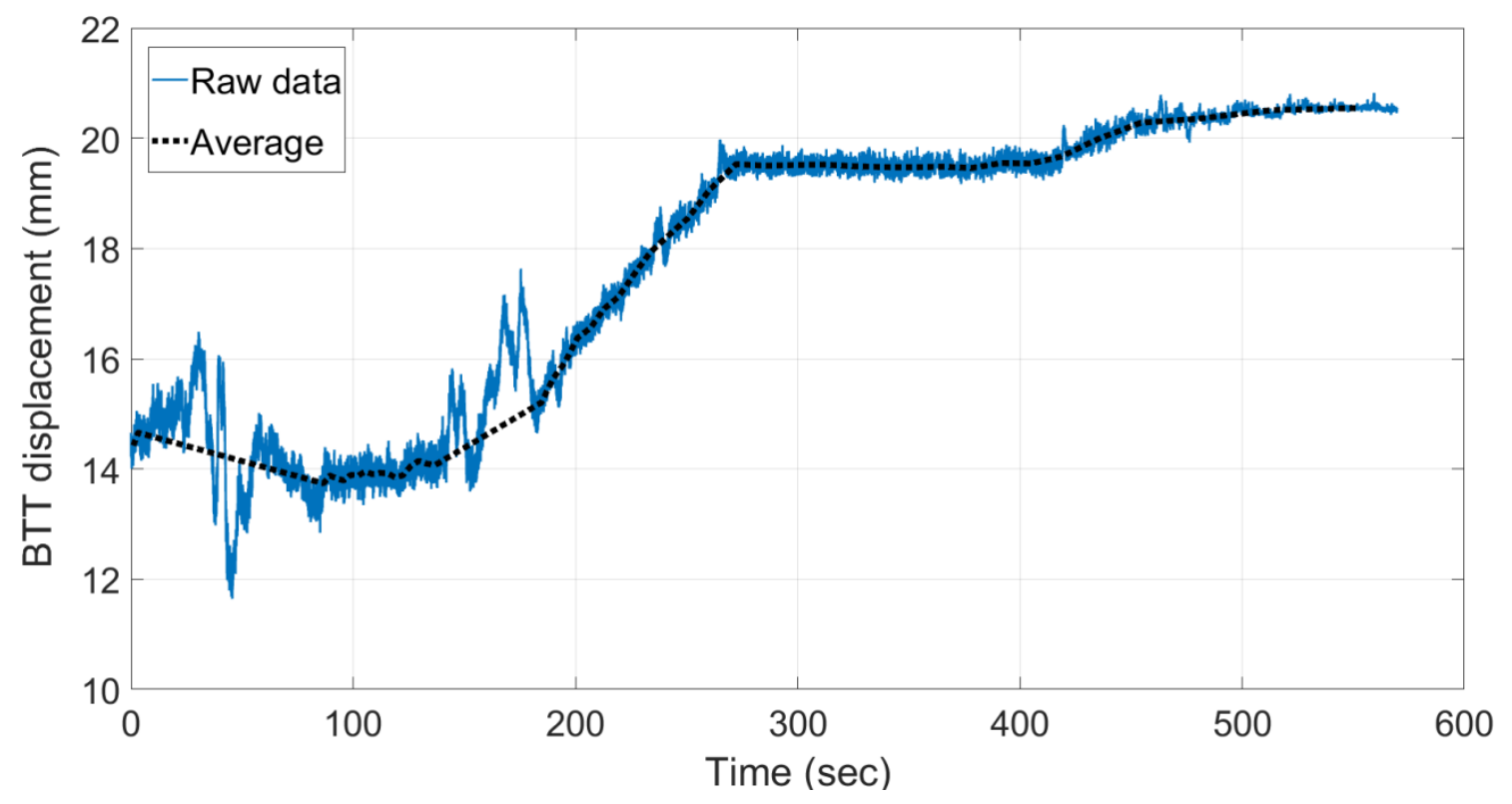

Fig. 28. Averaging the data of a single blade at probe 1 for the steady offset (as per section 4.1) for the second engine test. 


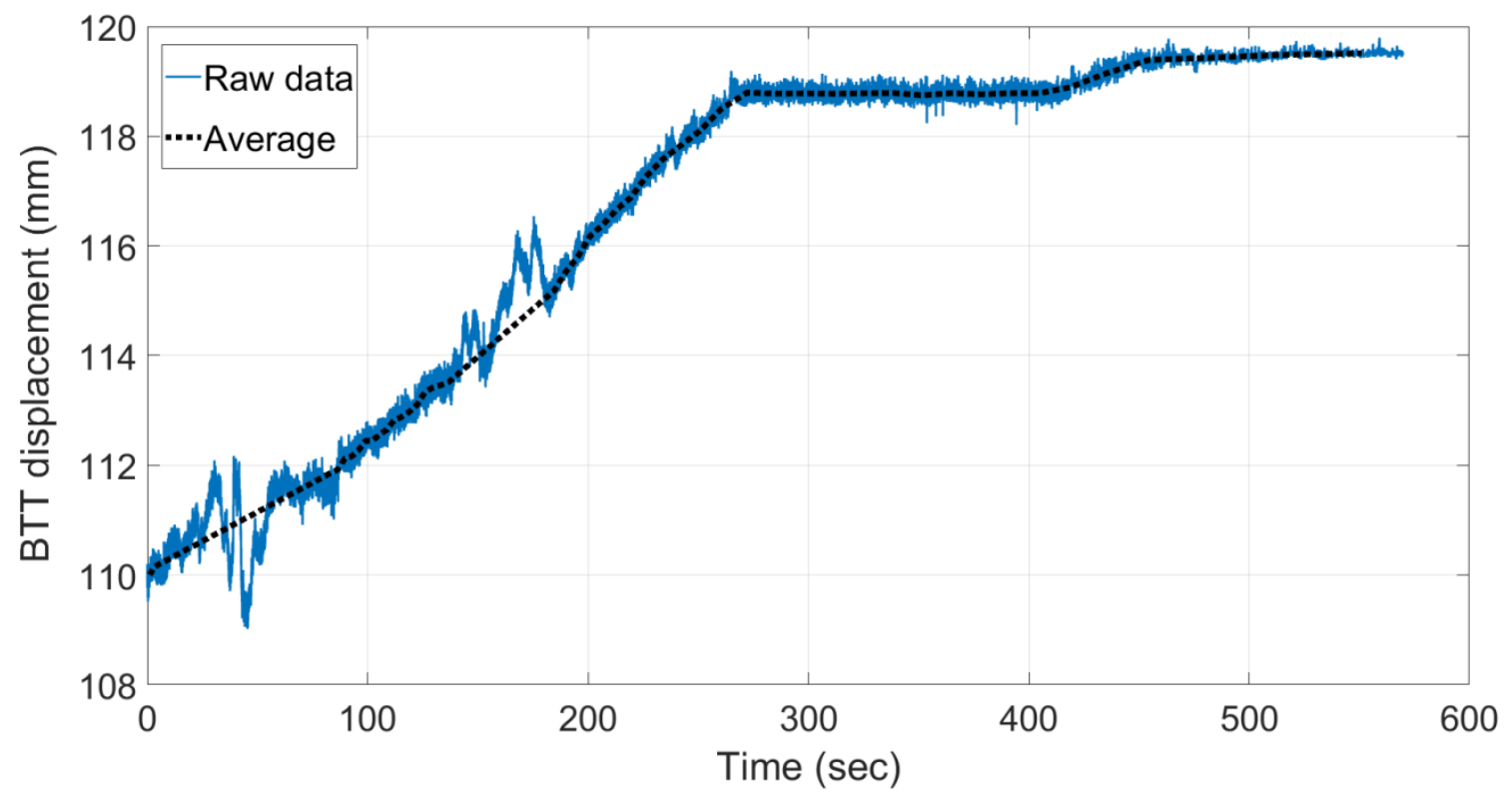

Fig. 29. Averaging the data of a single blade at probe 2 for the steady offset (as per section 4.1) for the second engine test.

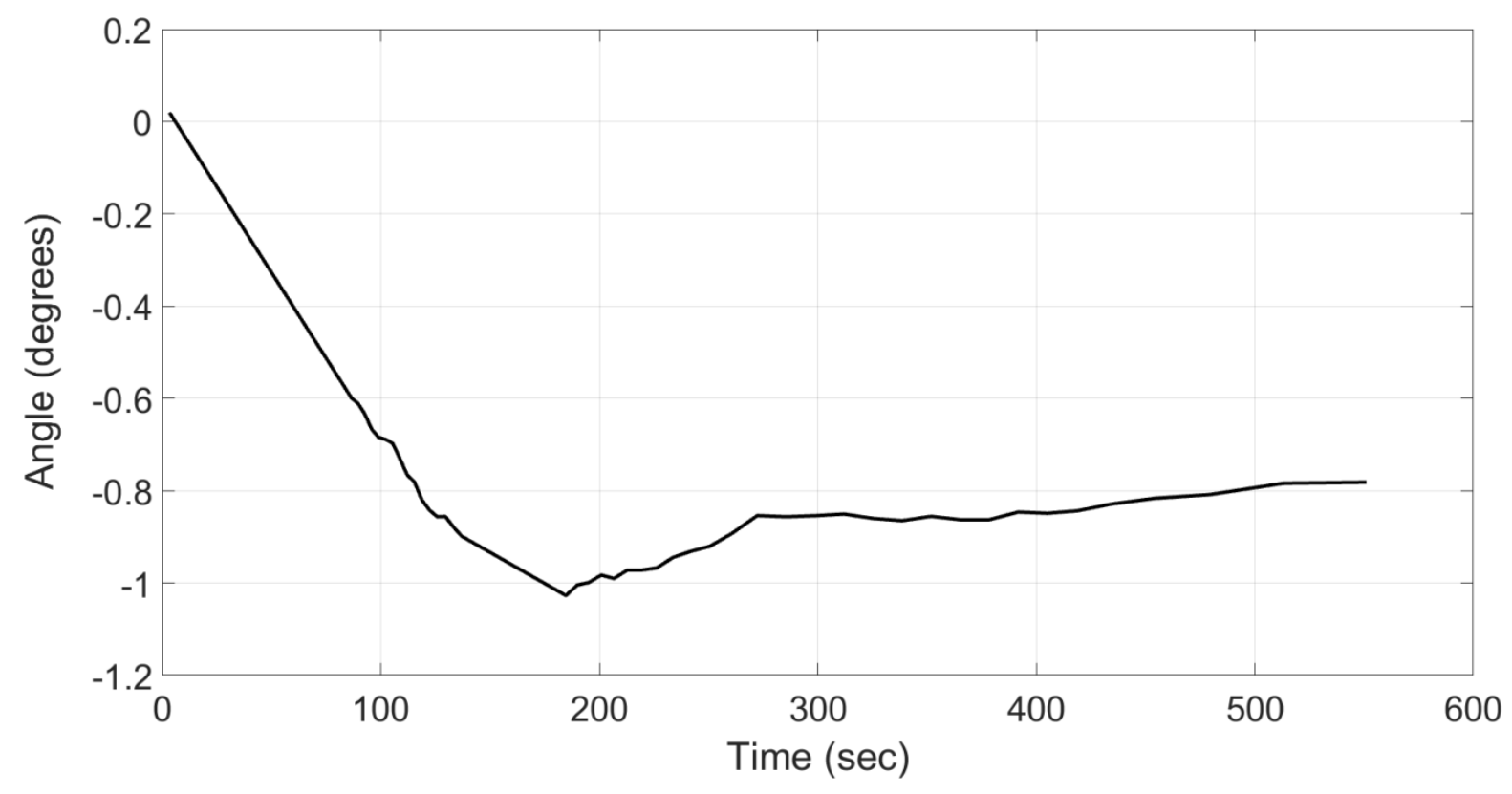

Fig. 30. Variation of the calculated untwist angle of a single blade for the second engine test. 